\title{
Optical Study of Active Ion Transport in Lipid Vesicles Containing Reconstituted Na,K-ATPase
}

\author{
H.-J. Apell ${ }^{\dagger}$, M.M. Marcus ${ }^{\dagger}$, B.M. Anner ${ }^{\ddagger}$, H. Oetliker ${ }^{\sharp}$, and P. Läuger ${ }^{\dagger}$ \\ 'Fakultät für Biologie, Universität Konstanz, D-7750 Konstanz. Federal Republic of Germany, Département de Pharmacologie, \\ Centre Médical Universitaire, Université de Genève. CH-I2II Genève 4, Switzerland, and \\ "Physiologisches Institut der Universität Bern, CH-3012 Bern. Switzerland
}

\begin{abstract}
Summary. A fluorescence method is described for the measurement of ATP-driven ion fluxes in lipid vesicles containing purified $\mathrm{Na}, \mathrm{K}$-ATPase. The membrane voltage of enzyme containing vesicles was measured by using a voltage-sensitive indocyanine dye. By addition of valinomycin the vesicle membrane is made selectively permeable to $\mathrm{K}^{+}$so that the membrane voltage approaches the Nernst potential for $\mathrm{K}^{+}$. With constant external $\mathrm{K}^{+}$ concentration, the time course of internal $\mathrm{K}^{+}$concentration can be continuously measured as change of the fluorescence signal after activation of the pump. The optical method has a higher time resolution than tracer-flux experiments and allows an accurate determination of initial flux rates. From the temperature dependence of active $\mathrm{K}^{+}$transport its activation energy was determined to be $115 \mathrm{~kJ} / \mathrm{mol}$. ATP-stimulated electrogenic pumping can be measured as a fast fluorescence change when the membrane conductance is low (i.e., at low or zero valinomycin concentration). In accordance with expectation, the amplitude of the fast signal change increases with decreasing passive ion permeability of the vesicle membrane. The resolution of the charge movement is so high that a few pump turnovers can be easily detected.
\end{abstract}

Key Words Na,K-ATPase reconstitution - potential sensitive dye - ion fluxes - transport kinetics - activation energy

\section{Introduction}

$\mathrm{Na}, \mathrm{K}-\mathrm{ATPa}$ e in the plasma membrane of mammalian cells transports sodium ions outward and potassium ions inward against their electrochemical potential gradients, utilizing ATP as energy source (Skou, 1975; for recent reviews, see Cantley, 1981; Schuurmans-Stekhoven \& Bonting, 1981; Jørgensen, 1982; Robinson, 1983). In the last years it has become possible to incorporate the purified enzyme into artificial phospholipid vesicles and to study its transport function in the reconstituted system (Goldin \& Tong, 1974; Hilden, Rhee \& Hokin, 1974; Racker \& Fisher, 1975; Anner, Lane, Schwartz \& Pitts, 1977; Rhoden \& Goldin, 1979; Anner, 1980;
Dixon \& Hokin, 1980; Jackson et al., 1980; Karlish \& Pick, 1981; Anner \& Moosmayer, 1982; Forgac \& Chin, 1982; Karlish, Lieb \& Stein, 1982; Abeywardena, Allen \& Charnock, 1983; Brotherus, Jacobsen \& Jørgensen, 1983; Cornelius \& Skou, 1984). Reconstituted vesicles prepared by cholate dialysis have diameters of about $90 \mathrm{~nm}$ and contain up to 10-20 pump molecules in random orientation (Skriver, Maunsbach, Anner \& Jørgensen, 1980a; Skriver, Maunsbach \& Jørgensen, 1980b; Anner, Robertson \& Ting-Beall, 1984b). Directionality of transport is achieved by adding ATP to the external medium, thereby activating only pump molecules with outward-facing ATP binding sites.

In the experiments with $\mathrm{Na}, \mathrm{K}$-ATPase vesicles carried out so far, the fluxes of $\mathrm{Na}^{+}$and $\mathrm{K}^{+}$have been studied with radioactive isotopes. The common version of this method requires separation of the vesicles from the external medium by filtration or gel chromatography and analysis of the radioactivity of the vesicles. The time resolution, which has been obtained by this technique, is limited by the sampling and separation procedure and is about a few seconds (Anner \& Moosmayer, 1982). A rapid sampling technique based on pressure filtration has recently been developed by Forbush (1984). This method yields sampling times of the order of 10 msec, but requires high pump densities in the membrane which are not easily achieved in reconstituted vesicles.

A sensitive method with a potentially high time resolution consists in monitoring optical signals associated with ion fluxes. In this communication we describe experiments in which a voltage-sensitive fluorescent dye, 1,3,3,1',3',3'-hexamethylindodicarbocyanine (Lüdi et al., 1983), is incorporated into the vesicle membrane. By addition of valinomycin the vesicle membrane is made selectively 
permeable to $\mathrm{K}^{+}$so that the membrane voltage becomes equal to the Nernst-potential for $\mathrm{K}^{+}$. The time course of fluorescence intensity after addition of ATP is a function only of the change of internal potassium concentration. Using a calibration of the fluorescence signal as a function of the $\mathrm{K}^{+}$concentration ratio, the rate of ATP-driven extrusion of $\mathrm{K}^{+}$ can be measured.

Under normal conditions, active ion transport by the $\mathrm{Na}, \mathrm{K}$-pump consists in a transport of two $\mathrm{K}^{+}$ and three $\mathrm{Na}^{+}$ions in opposite direction and therefore is associated with net movement of electric charge. This electrogenic action of the pump can be studied under conditions where the electrical resistance of the vesicle membrane is high, i.e., at low or zero ionophore concentration where the electrical effect of $\mathrm{Na}^{+}$uptake in excess of $\mathrm{K}^{+}$extrusion is not immediately cancelled by passive $\mathrm{K}^{+}$efflux. Under those conditions a fast change of the fluorescence signal is observed after ATP addition. This is interpreted as a change of membrane voltage produced by the pump current.

\section{Materials and Methods}

\section{MATERIALS}

Dioleoylphosphatidylcholine was obtained from Avanti Polar Lipids Inc., Birmingham, Ala.; sodium dodecylsulfate (SDS) from Pierce Chemical Company, Rockford, Ill., and sodium cholate from Merck, Darmstadt. Phosphoenolpyruvate, pyruvate kinase, lactate dehydrogenase, NADH and ATP (disodium salt, Sonderqualität) were from Boehringer, Mannheim. 1,3,3,1',3',3'-hexamethylindodicarbocyanine (NK 529):<smiles>CN1C(=CC=C=C=C2[N+](C)c3ccccc3C2(C)C)C(C)(C)c2ccccc21</smiles>

was purchased from Nippon Kankoh Shikiso Kenkyusho, Okayama, Japan. ${ }^{137} \mathrm{CsCl}$ was from New England Nuclear and ${ }^{22} \mathrm{NaCl}$ from Amersham International. All other reagents were obtained from Merck (analytical grade). Dialysis tubing was purchased from Serva, Heidelberg.

\section{BUFFER H}

If not otherwise indicated, the buffer for the vesicle experiments (buffer $\mathrm{H}$ ) contained $30 \mathrm{~mm}$ imidazol, $1 \mathrm{~mm}$ L-cystein, $1 \mathrm{~mm}$ ethylenediaminetetraacetic acid (EDTA), and $5 \mathrm{mM} \mathrm{MgSO}_{4}$; the $\mathrm{pH}$ was adjusted to 7.2 with $\mathrm{H}_{2} \mathrm{SO}_{4}$.

\section{EnZyme Preparation}

$\mathrm{Na}, \mathrm{K}-\mathrm{ATPase}$ was prepared from the outer medulla of rabbit kidneys using procedure $C$ of Jørgensen (1974). This method yields purified enzyme in the form of membrane fragments con- taining about $0.6 \mathrm{mg}$ phospholipid and $0.2 \mathrm{mg}$ cholesterol per $\mathrm{mg}$ protein (Jørgensen 1974, 1982). The specific ATPase activity was determined by the pyruvate kinase/lactatedehydrogenase assay (Schwartz et al. . 1971) and the protein concentration by the Lowry method (Lowry, Rosebrough, Farr \& Randall, 1951), using bovine serum albumin as standard. For most preparations the specific activity was in the range between 1500 and $2200 \mu \mathrm{mol} P_{i}$ per hr and $\mathrm{mg}$ protein at $37^{\circ} \mathrm{C}$. corresponding to a turnover rate of $120-170 \mathrm{~s}^{3}$ (based on a molar mass of $280,000 \mathrm{~g} / \mathrm{mol}$ )

\section{Na,K-ATPase Vesicles}

The purified Na,K-ATPase was solubilized in sodium cholate (Anner \& Moosmayer, 1981; Anner, Marcus \& Moosmayer, $1984 a) .2 \mathrm{mg}$ of the enzyme were suspended in $1 \mathrm{ml}$ "cholate buffer," consisting of buffer $\mathrm{H}$ with $23 \mathrm{~mm}$ sodium cholate and (if not otherwise stated) $70 \mathrm{mM} \mathrm{K}_{2} \mathrm{SO}_{4}$ plus $5 \mathrm{mM} \mathrm{Na}_{2} \mathrm{SO}_{4}$ (in most experiments $\mathrm{SO}_{4}^{2}$ was used as anion instead of $\mathrm{Cl}$ in order to minimize the passive anion permeation. The suspension was agitated with a high-speed Vortes mixer for $30 \mathrm{sec}$. After sedimentation in an Airfuge $(15 \mathrm{~min}$ at $100,000 \times g)$ the protein content and the enzyme activity of the supernatant were determined, yielding a recovery of about $40-50 \%$ of the protein content and activity before cholate addition.

A solution of dioleoylphosphatidylcholine in chloroform was evaporated under a stream of $\mathrm{N}_{2}$ in a round-bottom flask to yield a thin film on the glass wall. This procedure was twice repeated with ethyl ether. The lipid was solubilized by adding cholate buffer (see above) up to a concentration of $20 \mathrm{mg}$ lipid per $\mathrm{ml}$ and rotating the flask for $20 \mathrm{~min}$ at room temperature and for $2 \mathrm{~min}$ at $37^{\circ} \mathrm{C}$. Equal volumes of the resulting solution and the enzyme solubilisate were mixed, corresponding to a protein/lipid ratio of about $60 \mu \mathrm{g}$ protein per mg lipid. $200 \mu \mathrm{l}$ of the combined solubilisate were transfered to $7-\mathrm{mm}$ dialysis tubing and dialysed for $60 \mathrm{hr}$ at $4^{\circ} \mathrm{C}$ against $200 \mathrm{ml}$ buffer $\mathrm{H}$ containing (if not otherwise stated) $70 \mathrm{mM} \mathrm{K}_{2} \mathrm{SO}_{4}$ and $5 \mathrm{mM} \mathrm{Na}_{2} \mathrm{SO}_{4}$

Using egg phosphatidylcholine instead of dioleoylphosphatidylcholine and a similar preparation method, Skriver et al. $(1980 b)$ have shown that the dialysis yields single-shelled lipid vesicles with a diameter of about $90 \mathrm{~nm}$. The size distribution of the dioleoylphosphatidylcholine vesicles used in the experiments described below was determined by dynamic light scattering (Milsmann, Schwendener \& Weder, 1978); these measurements were kindly carried out by Dr. Schwendener at the ETH in Zürich. The average diameter of ATPase vesicles obtained by the light-scattering method was $96 \mathrm{~nm}$ and the half-width of the distribution $\pm 10 \mathrm{~nm}$. The diameter of protein-free vesicles was found to be $72 \mathrm{~nm}$ (half-width $\pm 0.8 \mathrm{~nm}$ ). The values of the halfwidth of the distribution are rough estimates, requiring further confirmation. The lipid content of the vesicle suspension after dialysis was determined by the phosphorous method (Bartlett, 1959) or by high-pressure liquid chromatography on a LiChrosorb Si 60 column (Knauer, Bad Homburg. F.R.G.) with: methanol/water as solvent. The loss of lipid during dialysis was found to be less than $10 \%$.

In order to determine the size of the entrapped aqueous space of the vesicles, ${ }^{137} \mathrm{Cs}^{+}$was added to the vesicle suspension. After variable time periods, the aqueous medium was separated from the vesicles by Sephadex G 50 gel chromatography and the radioactivity of the vesicles was counted (Anner, 1981). A period of $24 \mathrm{hr}$ was found to be sufficient for complete equilibration of ${ }^{137} \mathrm{Cs}^{+}$between vesicle interior and medium. From the radioactivity of the vesicles after equilibration, the entrapped volume was estimated to be $0.7 \%$ of the total volume of the suspension at a lipid concentration of $8 \mathrm{mg} / \mathrm{ml}$. This corresponds to an en- 
trapped volume $V$ of about $0.9 \mathrm{ml}$ per $\mathrm{g}$ lipid. (For comparison: the theoretical value of $V$ for spherical vesicles is given by $V=$ $(m / \sigma) /\left[\left(1+d / r_{i}\right)^{3}-1\right]$ where $m$ and $\sigma$ are the mass and the density of the lipid. $d$ the thickness of the membrane and $r_{i}$ the internal radius of the vesicle. With $\sigma=1 \mathrm{~g} / \mathrm{ml}, d=5 \mathrm{~nm}, r_{i}=40$ $\mathrm{nm}$, one obtains $V / \mathrm{m}=2.4 \mathrm{ml} / \mathrm{g}$. The fact that the experimental value of $\mathrm{V} / \mathrm{m}$ is two to three times smaller may result from the presence of leaky vesicles or from the formation of nonvesicular lipid aggregates.) Vesicle preparations used in fluorescence experiments were examined by tracer-flux assays with " $\mathrm{Na}$ and ${ }^{137} \mathrm{Cs}$ (instead of ${ }^{42} \mathrm{~K}$ ) to be in full agreement with published data (Anner. 1981: Anner et al., 1984a).

\section{Fluorescence Measurements AND DATA ANAI.YSIS}

The fluorescence measurements were carried out with a PerkinElmer 650-40 fluorescence spectrophotometer. The thermostated cell holder was equipped with a magnetic stirrer. The excitation wavelength was set to $620 \mathrm{~nm}$ (slit width $5 \mathrm{~nm}$ ) and the emission wavelength to $680 \mathrm{~nm}$ (slit width $20 \mathrm{~nm}$ ); for absorption and emission spectra of the indocyanine dye, see Lüdi, Oetliker and Brodbeck (1981). A $25 \%$ grey filter was used on the excitation side. The cell was filled with $1 \mathrm{ml}$ of buffer $\mathrm{H}$ containing (if not otherwise stated) $5 \mathrm{mM} \mathrm{K}_{2} \mathrm{SO}_{4}, 70 \mathrm{mM} \mathrm{Na}_{2} \mathrm{SO}_{4}$ and $2.3 \mu \mathrm{M}$ $1,3,3,1^{\prime}, 3^{\prime}, 3^{\prime}$-hexamethylindodicarbocyanine. The dye was added from a $0.1 \%(\mathrm{wt} / \mathrm{vol})$ stock solution in $1: 9(\mathrm{vol} / \mathrm{vol})$ ethanol/water. $5 \mu \mathrm{l}$ of the vesicle suspension containing approximately $10 \mathrm{mg} \mathrm{lipid} / \mathrm{ml}$ was added to the solution in the cell. After the fluorescence signal reached a steady value, valinomycin was added from a concentrated stock solution in ethanol. Addition of the same amount of ethanol had no effect on the fluorescence. The solution in the cell was continuously stirred. The time resolution of the measurement was limited by the mixing time after reagent addition to the cell and was about $3 \mathrm{sec}$. The solution in the cell was kept at a temperature of $16^{\circ} \mathrm{C}$ (if not otherwise stated). Superimposed onto the fluorescence signal resulting from changes of membrane voltage was a steady downward drift of the signal of $\leqslant 0.4 \%$ per min, probably resulting from a slow aggregation of the dye in the aqueous medium. (A similar drift is observed when dye is added to the solution in the absence of vesicles.) The magnitude of the drift was determined before the addition of ATP and at the end of the experiment. All fluorescence data were corrected for the average magnitude of the drift.

As described in the next section, the relative fluorescence signal $\Delta F / F_{\text {o }}$ was calibrated as a function of membrane voltage $U$ by measuring $\Delta F / F_{o}$ at different $\mathrm{K}^{+}$concentrations in the medium in the presence of valinomycin. A smooth curve was fitted to the observed $\Delta F / F_{o}$, values plotted as a function of Nernst potential $E_{K}=U$. This curve was digitized and used in data processing programs on a MINC $11 / 23$ computer. In the analysis of the flux experiments, a linear interpolation procedure was used for calculating $U$ from the experimental $\Delta F / F_{o}$, values and the calibration data. Fluorescence/time curves were digitized from the chart recorder output and stored on disk for further analysis such as signal averaging or calculation of time derivatives.

\section{Results}

\section{Calibration of the Fluorescence Signals}

In order to calibrate the fluorescence signal as a function of membrane voltage $U$, the method of

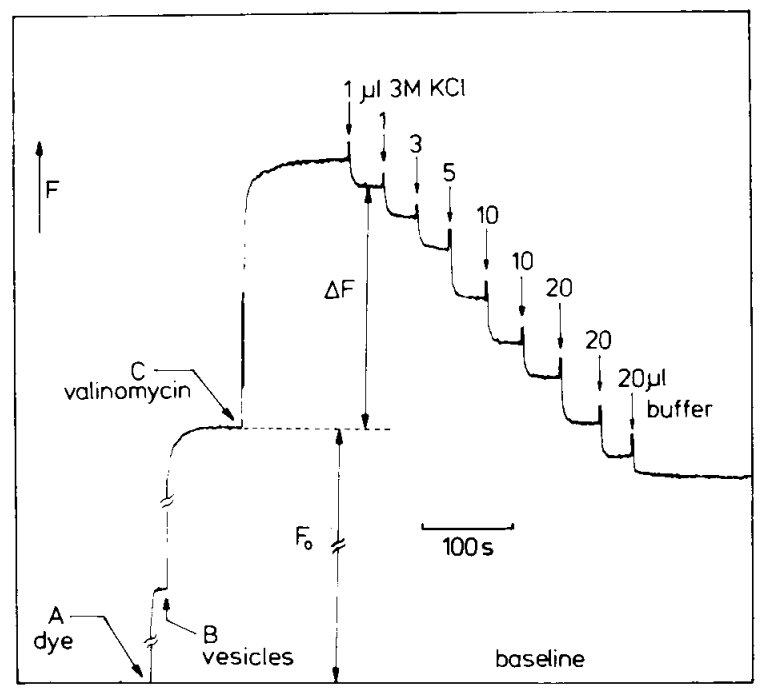

Fig. 1. Variation of fluorescence intensity with external potassium concentration. $F$ is the fluorescence signal in arbitrary units. At the start of the experiment $1 \mathrm{ml}$ buffer $\mathrm{H}$ containing 5 $\mathrm{mM} \mathrm{K}_{2} \mathrm{SO}_{4}$ and $70 \mathrm{mM} \mathrm{Na} \mathrm{SO}_{4}$ was present in the fluorescence cell. Thereafter the following additions were made; $A: I \mu \mathrm{l}$ stock solution (2.3 $\mathrm{mm}$ ) of $1,3,3,1^{\prime}, 3^{\prime}, 3^{\prime}$-hexamethylindodicarbocyanine; $B: 10 \mu \mathrm{l}$ vesicle suspension ( $10 \mathrm{mg}$ lipid per $\mathrm{ml}$ ) in buffer $\mathrm{H}$ containing $70 \mathrm{mM} \mathrm{K}_{2} \mathrm{SO}_{4}$ and $5 \mathrm{mM} \mathrm{Na}_{2} \mathrm{SO}_{4} ; C: 5 \mu \mathrm{l}$ stock solution $(4 \mu \mathrm{M})$ of valinomycin: $I, 3,5,10,20: 1 \mu \mathrm{l}, 3 \mu \mathrm{l}, 5$ $\mu \mathrm{l}, 10 \mu \mathrm{l}$ and $20 \mu \mathrm{l}$, respectively, of a $3 \mathrm{M} \mathrm{KCl}$ solution. The signal change upon addition of buffer solution (buffer $\mathrm{H}$ ) results from the dilution of the dye. The baseline corresponds to the signal prior to the addition of the dye. Addition of vesicles without dye gave a very small positive signal $\left(<1 \%\right.$ of $\left.F_{6}\right)$. Addition of valinomycin (up to a total concentration of $20 \mathrm{nM}$ ) to the vesicles at zero $\mathrm{K}^{+}$-gradient $\left(c_{\mathrm{K}}^{e}=c_{\mathrm{K}}^{i}\right)$ gave only an insignificant fluorescence change $\left(\Delta F / F_{0}<0.08\right) . T=16^{\circ} \mathrm{C}$

Hoffman and Laris (1974) was used in which a gradient of potassium concentration $c_{\mathrm{K}}$ is maintained across the vesicle membrane in the presence of the potassium-ionophore valinomycin. Under this condition the membrane voltage $U$ is equal to the Nernst potential for potassium:

$U \equiv \psi_{i}-\psi_{e}=\frac{R T}{F} \ln \frac{c_{\mathrm{K}}^{e}}{c_{\mathrm{K}}^{i}}$

$R$ is the gas constant, $T$ the absolute temperature, $F$ the Faraday constant, and $\psi_{i}, \psi_{e}, c_{\mathrm{K}}^{i}, c_{\mathrm{K}}^{e}$ are the electric potentials and the potassium concentrations in the internal and external medium.

A record of a calibration experiment is represented in Fig. 1. When vesicles were added to the buffer solution containing the indocyanine dye, an increase of fluorescence intensity was observed, which presumably resulted from the change of polarity in the vicinity of the dye molecule upon binding to the membrane. Under the conditions of the 


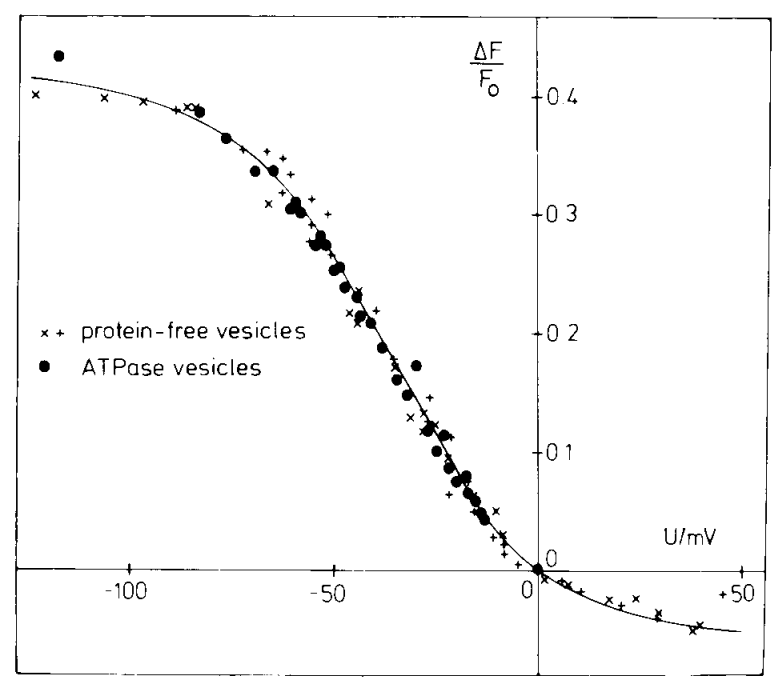

Fig. 2. Calibration of the fluorescence signals as a function of membrane voltage $U . F_{0}$ is the fluorescence signal prior to the addition of valinomycin (Fig. 1) and $\Delta F$ is the fluorescence incre ment in the presence of valinomycin at a given ratio $c_{\mathrm{K}}^{\prime \prime} / c_{\mathrm{K}}^{i}$ of the external and internal potassium concentrations. The membrane voltage $U$ was calculated from $c_{\mathrm{K}}^{k}$ and $c_{\mathrm{K}}^{i}$ using the Nernst relation [Eq. (1)]. $\times$ and + , protein-free vesicles formed in buffer $\mathrm{H}$ containing $75 \mathrm{mM}\left(\mathrm{K}_{2} \mathrm{SO}_{4}+\mathrm{Na}_{2} \mathrm{SO}_{4}\right)$ in various $\mathrm{K}^{+} / \mathrm{Na}^{+}$ratios. The vesicles were added to (iso-osmolar) buffer $\mathrm{H}$ containing 75 $\mathrm{mM}\left(\mathrm{K}_{2} \mathrm{SO}_{4}+\mathrm{Na}_{2} \mathrm{SO}_{4}\right)$ in different $\mathrm{K}^{+} / \mathrm{Na}^{+}$ratios; data points indicated by + refer to experiments in which $c_{\mathrm{K}}^{\prime} / c_{\mathrm{K}}^{i}$ was further varied by adding small volumes of $3 \mathrm{M} \mathrm{KCl}$ to the medium (Fig. $1)$; in this case $\Delta F$ was corrected for the dilution of the dye and $c_{\mathrm{K}}^{i}$ for the osmotic loss of water from the vesicle interior (see text). $\bullet$, vesicles containing $\mathrm{Na}, \mathrm{K}-\mathrm{ATPase} ; c_{\mathrm{K}}^{\mathrm{e}} / c_{\mathrm{K}}^{\mathrm{c}}$ was varied as in the experiments marked by + . The temperature was $16^{\circ} \mathrm{C}$ throughout

experiment (see legend of Fig. 1) the initial potassium concentrations were $140 \mathrm{~mm}$ in the vesicle interior and $10 \mathrm{~mm}$ in the medium. Addition of valinomycin led to a further increase of fuorescence intensity, which is thought to reflect the development of the Nernst potential for $\mathrm{K}^{+}$. The initial value of the membrane voltage, according to Eq. (1), was $U=-66 \mathrm{mV}$. When the $\mathrm{K}^{+}$concentration gradient was diminished by adding small volumes $(1-20 \mu \mathrm{l})$ of $3 \mathrm{M} \mathrm{KCl}$ solution to the medium, the fluorescence signal decreased again. In this way the fluorescence intensity was determined for different values of the concentration ratio $c_{\mathrm{K}}^{\mathrm{e}} / c_{\mathrm{K}}^{i}$. Only a small fraction of the fluorescence change resulted from the dilution of the dye in the cell; the magnitude of the dilution effect was determined by addition of small volumes of buffer solution. Furthermore, possible effects from changes in the light scattering of the vesicles could be excluded, since the signal which was recorded from a vesicle sus- pension without dye was less than $1 \%$ of the signal in the presence of dye.

In Fig. 2 the ratio $\Delta F / F_{o}$ is plotted as a function of the calculated Nernst potential. $F_{0}$, is the fluorescence signal prior to the addition of valinomycin, and $\Delta F$ the fluorescence increment in the presence of valinomycin at a given ratio $\left(c_{\mathrm{K}}^{i} / c_{\mathrm{K}}^{i}\right.$ of the potassium concentrations (Fig. 1). $\left(c_{\mathrm{K}}^{i} / c_{\mathrm{K}}^{i}\right.$ was varied by forming the vesicles in buffer solutions containing $\mathrm{K}^{+}$and $\mathrm{Na}^{+}$and by diluting the vesicle suspension into an iso-osmolar solution of different $\mathrm{K}^{+} / \mathrm{Na}^{+} \mathrm{ra}-$ tio. When a given ratio $\left(c_{\mathrm{K}} / c_{\mathrm{K}}^{i}\right.$ was generated using solutions of different absolute values of $c_{\mathrm{K}}^{i}$ and $c_{\mathrm{K}}^{i}$, the results were virtually identical. In some experiments $c c_{\mathrm{K}}^{\prime} / c_{\mathrm{K}}^{i}$ was varied by adding small volumes of $3 \mathrm{~m} \mathrm{KCl}$ to the medium, as described above (compare Fig. 1). In latter experiments in which the osmolarity of the medium changed upon addition of concentrated $\mathrm{KCl}$ solution, $c_{\mathrm{K}}^{i}$ was corrected for the osmotic loss of water from the vesicle interior. (For a spherical vesicle of radius $r$, the time constant $\tau$ for the establishment of osmotic equilibrium is of the order of $r / 3 P_{n}$ where $P_{n}$ is the permeability coefficient of water. With $P_{n}=\left(10^{-4}-10^{3}\right) \mathrm{cm} / \mathrm{sec}$ (Lawaczek, 1979; Carruthers \& Melchior, 1983) and $r$ $=45 \mathrm{~nm}, \tau$ becomes about $1-10 \mathrm{msec}$, meaning that osmotic equilibrium is always established within the time scale of these experiments.) It is seen from Fig. 2 that the two methods of varying $\left(c_{\mathrm{K}} / c_{\mathrm{K}}^{i}\right.$ gave essentially the same results. The relationship between $\Delta F / F_{o}$ and $U$ was found to be virtually identical for protein-free vesicles and vesicles containing $\mathrm{Na}, \mathrm{K}$-ATPase. Furthermore, the $\left(\Delta F / F_{0}\right.$ vs. $\left.U\right)$ curve did not significantly depend on temperature between 6 and $22^{\circ} \mathrm{C}$.

As shown by Fig. 2, the voltage-dependence of $\Delta F / F_{0}$ is strongly asymmetric. At inside-negative voltages, $\Delta F / F_{0}$ is a linear function of $U$ in the range between -60 and $-10 \mathrm{mV}$, whereas for $U>0$ the fluorescence signal approaches a limiting value $\Delta F / F_{0} \simeq-0.054$. In the linear range the relative fluorescence change is about $6 \%$ per $10 \mathrm{mV}$ voltage change. The fact that $\Delta F$ goes through zero at $U \approx 0$ means, of course, that the fluorescence in the absence of valinomycin under the condition $c_{\mathrm{K}}^{\prime} \neq c_{\mathrm{K}}^{i}$ is nearly the same as the fluorescence in the presence of valinomycin under the condition $c_{\mathrm{K}}^{\prime \prime}=c_{\mathrm{K}}^{i}$. This could mean that the intrinsic permeabilities of the vesicle membrane for $\mathrm{K}^{+}$and $\mathrm{Na}^{+}$are almost identical (Anner, 1981), or that the membrane potential in the absence of valinomycin is determined by an ion species present in identical concentrations on both sides, for instance imidazol- $\mathrm{H}^{+}$.

In experiments with squid giant axons, Ross et al. (1977) observed that the sign of the fluorescence change for a given polarity of the voltage signal was 
the same, irrespective of whether the dye was added to the medium or injected into the axoplasm. They concluded that the membrane was relatively permeable for the dye and that its intramembrane adsorption sites could be reached from either side of the membrane. This symmetry of the sign of $\Delta F$ in the squid-axon experiments is remarkable in view of the strongly asymmetric behavior of $\Delta F / F_{0}$ in the vesicle experiments (Fig. 2). A reason for this difference could be that the dye cannot permeate the vesicle membrane as easily as the axon membrane. Another possibility consists in the assumption that differences in the structure of the membrane surface resulting from the finite radius of curvature $(\sim 45 \mathrm{~nm})$ lead to an asymmetry in dye adsorption.

For the establishment of the Nernst potential for $\mathrm{K}^{+}$, the valinomycin-induced $\mathrm{K}^{+}$permeability must be sufficiently high in order to overcome the intrinsic permeabilities of the other ions present in the system. An experiment in which the valinomycin concentration $c_{v}$ was varied is represented in Fig. 3. It is seen that $\Delta F / F_{0}$, approaches a limiting value for $c_{v} \geqslant 10 \mathrm{~nm}$. Accordingly, a valinomycin concentration of $20 \mathrm{nM}$ was used in the Nernstpotential measurements. (At larger values of $c_{v}$, valinomycin alone, i.e., in the absence of a potassium-concentration gradient, produces a small fluorescence change which presumably results from a direct interaction between dye and valinomycin in the membrane.)

If $\gamma$ is the lipid/water partition coefficient of (uncomplexed) valinomycin and $V_{l}$ and $V_{w}$ are the volumes of the lipid and the water phase, respectively, the fraction $\theta$ of valinomycin bound to the lipid is given by

$\theta \equiv \frac{n_{l}}{n_{l}+n_{w^{*}}}=\frac{\gamma V_{l} / V_{w^{\prime}}}{1+\gamma V_{l} / V_{w}}$

$n_{l}$ and $n_{w}$ denote the number of moles of valinomycin in the lipid and the water phase. For a lipid concentration of $0.1 \mathrm{mg} / \mathrm{ml}$ in the aqueous phase, the ratio $V_{l} / V_{w}$ is approximately $10^{-4}$. With a partition coefficient $\gamma \simeq 5 \times 10^{4}$ (Benz, Stark, Janko \& Läuger, 1973), $\theta$ becomes equal to 0.8 . Under the usual experimental conditions ( $20 \mathrm{~nm}$ valinomycin, $0.1 \mathrm{mg} \mathrm{lipid} / \mathrm{ml}$ ) about $0.2 \mu \mathrm{mol}$ valinomycin is present in the membrane per $g$ lipid. Since a unilamellar lecithin vesicle of $96 \mathrm{~nm}$ external diameter contains $1.1 \times 10^{-16} \mathrm{~g}$ lipid, the number of valinomycin molecules per vesicle is about 10 . A spherical vesicle of this diameter has a membrane capacitance of $\mathrm{C} \simeq 2 \times 10^{-16} \mathrm{~F}$ (assuming a specific capacitance of $1 \mu \mathrm{F} / \mathrm{cm}^{2}$ ). At this value of $C$ about 100 univalent ions have to move across the membrane in order to build up a Nernst potential of $100 \mathrm{mV}$;

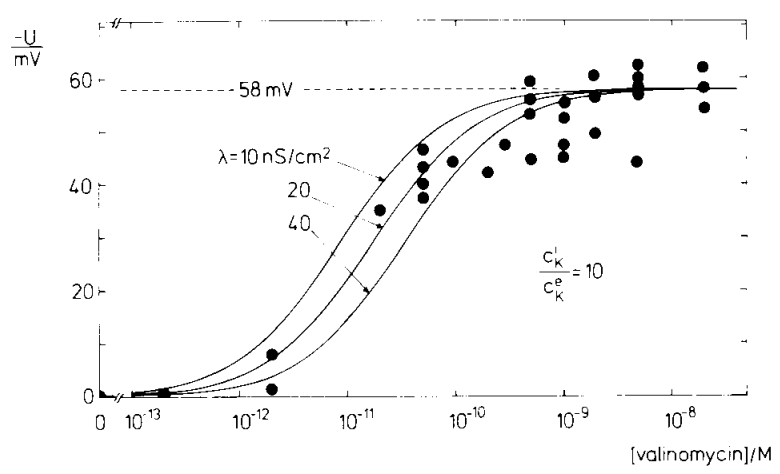

Fig. 3. Membrane voltage $U$ as a function of valinomycin concentration at a constant $\mathrm{K}^{+}$concentration ratio $c_{\mathrm{K}}^{i} / c_{\mathrm{K}}^{e}=10 . U$ was obtained from the fluorescence signals $\Delta F / F_{o}$ using the calibration given in Fig. 2. The lines represent theoretical curves drawn according to Eq. (18) with $\alpha=2.6 \times 10{ }^{13} \mathrm{M}^{2}$ (left), $5.2 \times$ $10{ }^{13} \mathrm{M}^{2}$ (middle) and $10.4 \times 10^{1.3} \mathrm{M}^{2}$ (right). With a $\mathrm{K}^{+}$permeability coefficient $P_{\mathrm{K}}=1 \times 10^{7} \mathrm{~cm} / \mathrm{sec}$ at $c_{i}=10 \mathrm{nM}$, the corresponding values of the leakage conductance become $\lambda=10$ $\mathrm{nS} / \mathrm{cm}^{2}, 20 \mathrm{nS} / \mathrm{cm}^{2}$ and $40 \mathrm{nS} / \mathrm{cm}^{2}$, respectively $\mid$ Eqs. (17) and (18)]. Na,K-ATPase vesicles were formed in buffer $\mathrm{H}$ containing $140 \mathrm{~mm} \mathrm{KCl}$ and $10 \mathrm{~mm} \mathrm{NaCl} .5 \mu$ lof the vesicle suspension $(10$ $\mathrm{mg}$ lipid $/ \mathrm{ml}$ ) were diluted into $1 \mathrm{ml}$ buffer $\mathrm{H}$ containing $10 \mathrm{mM}$ $\mathrm{KCl}, 140 \mathrm{~mm} \mathrm{NaCl}$ and $2.3 \mu \mathrm{M}$ indocyanine dye

this is a negligible fraction (about $0.6 \%$ ) of the total $\mathrm{K}^{+}$content of the vesicle at $c_{\mathrm{K}}^{i}=0.1 \mathrm{M}$. At a potassium concentration of $0.1 \mathrm{M}$ the turnover rate of valinomycin in a lecithin membrane is of the order of $100 \mathrm{~s}^{-1}$ (Benz et al., 1973). Under the given experimental conditions the time constant for the establishment of the Nernst potential may be estimated to be about 10 turnover times, or $0.1 \mathrm{sec}$, which is below the time resolution of the used setup. If, however, valinomycin is added in lower concentrations, the fluorescence signal changes with a measurable time constant of about $80 \mathrm{sec}$ at $c_{v}=0.1 \mathrm{nM}$. At this concentration the average number of valinomycin molecules per vesicle is estimated to be about 0.05 . The observed time-delay may result from a slow exchange of valinomycin molecules between lipid vesicles via the aqueous phase.

\section{ATP-Driven Extrusion of $\mathrm{K}^{+}$}

The result of an experiment in which the $\mathrm{Na}, \mathrm{K}$ ATPase was activated by addition of ATP is shown in Fig. $4 A$. The vesicles initially contained $140 \mathrm{~mm}$ $\mathrm{K}^{+}$, the medium $10 \mathrm{~mm} \mathrm{~K}^{+}$and $20 \mathrm{~nm}$ valinomycin. At this valinomycin concentration the membrane voltage may be assumed to be equal to the Nernst potential for $\mathrm{K}^{+}$. After addition of ATP to the medium the fluorescence starts to decrease. This fluo- 

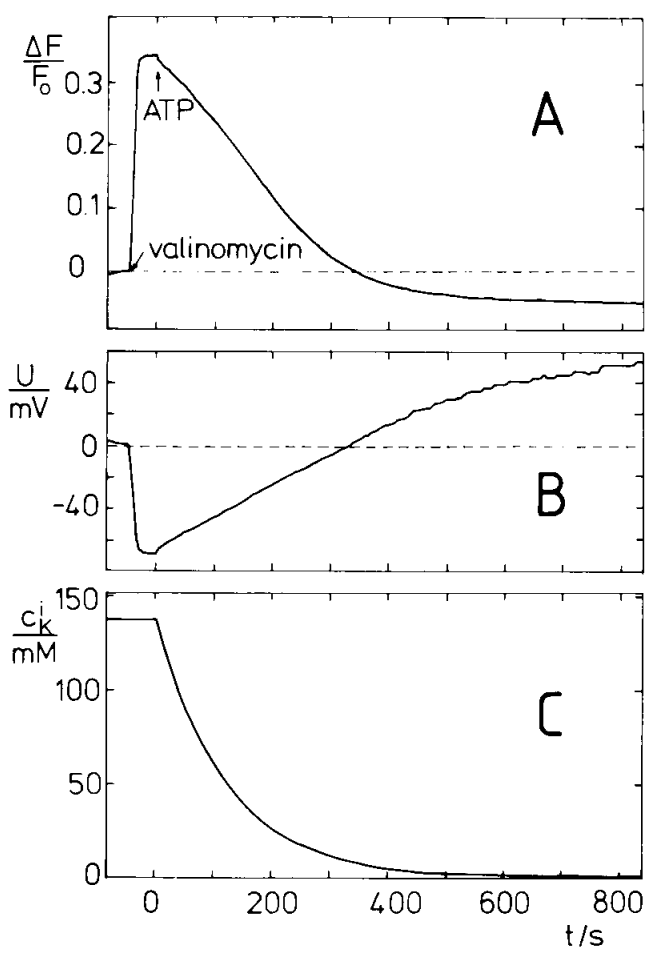

Fig. 4. ATP-driven extrusion of $\mathrm{K}^{+}$at $16^{\circ} \mathrm{C}$. Na, K-ATPase vesicles were formed in buffer $\mathrm{H}$ containing $70 \mathrm{mM} \mathrm{K}_{2} \mathrm{SO}_{4}$ and $5 \mathrm{~mm}$ $\mathrm{Na}_{2} \mathrm{SO}_{4} 5 \mu \mathrm{l}$ of the vesicle suspension $(10 \mathrm{mg} \mathrm{lipid} / \mathrm{ml}$ ) were diluted into $1 \mathrm{ml}$ buffer $\mathrm{H}$ containing $5 \mathrm{mM} \mathrm{K}_{2} \mathrm{SO}_{4}$ and $70 \mathrm{~mm}$ $\mathrm{Na}_{2} \mathrm{SO}_{4}$. Thereafter, $2.3 \mu \mathrm{M}$ indocyanine dye, $20 \mathrm{nM}$ valinomycin and $250 \mu \mathrm{M}$ ATP were added successively. $(A)$ : fluorescence signal $\Delta F$, divided by the signal $F_{o}$ prior to the addition of valinomycin (compare Fig. 1). $\Delta F / F_{o}$, has been corrected for the initial dilution effect and for a steady drift of $\sim 8 \times 10^{-5} \mathrm{~s}^{-1}$. (B): membrane voltage $U$ as obtained from $\Delta F / F_{0}$ and the calibration curve (Fig. 2). (C): Internal potassium concentration $c_{\mathrm{K}}^{i}(t)$ as calculated from $U(t)$ using the Nernst relation under the assumption that $c_{\mathrm{K}}^{*}$ remains virtually constant. $c_{\mathrm{K}}^{i}$ values below $10 \mathrm{~mm}$ are uncertain since for $U>0$ the fluorescence signal $\Delta F$ is rather insensitive to variations of $U$ (compare Fig. 2). Virtually identical results were obtained using an alternative calibration procedure based on the difference $Y(t) \equiv F(t)-F(x)$, where $F(t)$ is the fluorescence signal at time $t$ (corrected for signal drift). Assuming that $F(0)$ corresponds to the initial Nernst voltage $U_{0}$ and $F(\infty)$ to the saturation value of the fluorescence signal at large positive voltages (Fig. 2), the voltage $U$ was obtained from the calibration curve $U(X)$ by putting $X=|Y(t) / Y(0)| \cdot X\left(U_{0}\right)$. The quantity $X$ is defined as the difference of the fluorescence signals $F_{\text {, in }}$ in the calibration experiment at the Nernst potential $U$ minus the limiting $F_{c}$ value for $U \gg R T / F$ : $X(U) \equiv F_{c}(U)-F_{c}(x)$

rescence change is thought to reflect the decrease of internal $\mathrm{K}^{+}$concentration, which results from the activation of pump molecules oriented with their ATP binding sites facing outward. Since the vesicle membrane has a high $\mathrm{K}^{+}$permeability in the presence of $20 \mathrm{~nm}$ valinomycin, any net charge trans- port by the pump is compensated by a passive counterflow of $\mathrm{K}^{+}$. This means that the total (ATP-driven plus passive) $\mathrm{K}^{+}$efflux is equal to the ATP-driven $\mathrm{Na}^{+}$influx. ATP was added to a final concentration of $250 \mu \mathrm{M}$. The estimated amount of ATP needed to pump all $\mathrm{K}^{+}$ions out of the vesicles was less than $1 \%$ of the available ATP.

In the experiment represented in Fig. $4 A$ the $\mathrm{K}^{+}$ concentration ratio $c_{\mathrm{K}}^{i} / c_{\mathrm{K}}^{\prime \prime}$ was 14 , corresponding (at $16^{\circ} \mathrm{C}$ ) to a Nernst-potential of $-66 \mathrm{mV}$. Experimental conditions with $c_{\mathrm{K}}^{i}>c_{\mathrm{K}}^{i}$ were chosen because externally added indocyanine dye exhibits optimum voltage sensitivity for inside-negative potentials (Fig. 2). Since $\mathrm{K}^{+}$is close to electrochemical equilibrium and $\mathrm{Na}^{+}$is transported downhill, the pump operates in a purely dissipative mode. Experiments with initially symmetrical concentrations $\left(c_{K}^{i}=c_{K}^{i}\right)$ were also carried out. A fluorescence decrease of the expected (low) amplitude was observed after addition of ATP. In this case an inside-positive membrane voltage develops, so that the fluorescence signal soon reaches a saturation level.

When ATP was added to a suspension of protein-free vesicles under otherwise identical conditions as in the experiment of Fig. 4, only a small fluorescence change corresponding to the dilution of the dye was observed. A similar control experiment was carried out with $\mathrm{Na}, \mathrm{K}$-ATPase vesicles (Fig. 5). After addition of ATP in the absence of $\mathrm{Mg}^{2+}$, the fluorescence remained constant (apart from the dilution effect). When thereafter $\mathrm{Mg}^{2+}$ was added, the fluorescence intensity started to decline to a low level with a similar time course as in Fig. 4. These findings are consistent with the known requirement of the $\mathrm{Na}, \mathrm{K}-\mathrm{ATPase}$ for $\mathrm{Mg}^{2+}$ (Jørgensen, 1982) and demonstrate that ATP per se does not have any effect on the membrane voltage. $\mathrm{Mg}^{2+}$ alone has also no effect on the membrane voltage.

From the $\Delta F / F_{o}$ values taken from Fig. $4 A$, the membrane voltage $U$ as a function of time $t$ may be evaluated using the calibration curve (Fig. 2) for $\Delta F / F_{o}$. The result is represented in Fig. $4 B$. Since the volume ratio $V_{e} / V$ of external and internal space is about $1000, c_{\mathrm{K}}^{e}$ remains virtually constant during the flux experiment. The time course of the internal $\mathrm{K}^{+}$concentration $c_{\mathrm{K}}^{i}$ is then directly obtained from $U(t)$ and the Nernst relation [Eq. (1)]. As seen from Fig. $4 C, c_{\mathrm{K}}^{i}$ decreases to one half of the initial value within about $80 \mathrm{sec}$. $c_{\mathrm{K}}^{i}$ values below $10 \mathrm{~mm}$ are uncertain since for $U>0$ the fluorescence signal is rather insensitive to variations of $U$ (compare Fig. 2).

If $\nu \mathrm{Na}^{+}$ions are transported inward per pump cycle, an equal number of $\mathrm{K}^{+}$ions must leave the vesicle under the condition of high passive $\mathrm{K}^{+}$permeability, as discussed above. The time derivative 
of $c_{\mathrm{K}}^{i}$ is related to the turnover rate $v$ of the pump and the average number $n_{P}$ of active pump molecules per vesicle (ATP binding site facing outward):

$\nu n_{P} v=-V \frac{d c_{\mathrm{K}}^{i}}{d t}$

$V$ is the average volume of the entrapped aqueous phase of a vesicle. For a spherical vesicle of external radius $r_{p^{\prime}} \simeq 45 \mathrm{~nm}$ and membrane thickness $d \simeq 5$ $\mathrm{nm}$, the entrapped volume is $V \simeq 2.7 \times 10^{-4} \mu \mathrm{m}^{3}$. Assuming $\nu=3$ and taking $d c_{\mathrm{K}}^{i} / d t$ at time zero from Fig. $4 C$, the initial pump rate $n_{P} v_{0}$, per vesicle is estimated to be $80 \mathrm{~s}^{-1}$.

It is seen from Fig. $4 C$ that $d c_{\mathrm{K}}^{i} / d t$ decreases with time. According to Eq. (3) this would mean that the transport rate $v$ decreases, since $\nu, n_{P}$ and $V$ are likely to remain constant during the experiment. A decrease of $v$ has to occur since $\mathrm{K}^{+}$is pumped out from the vesicles, but the observed rate of decay is much larger than expected. $d c_{\mathrm{K}}^{i} / d t$ in Fig. $4 C$ has declined to half the initial value when the $\mathrm{K}^{+}$concentration in the vesicles is still $65 \mathrm{~mm}$. The $\mathrm{K}_{\mathrm{m}}$ value of the pump for $\mathrm{K}^{+}$, however, is much smaller, of the order of $0.1 \mathrm{~mm}$ (Robinson \& Flashner, 1979). Likewise, it is improbable that the decrease of $d c_{\mathrm{K}}^{i} / d t$ results from an effect of membrane voltage $U$ on pump rate, since the variation of voltage $U$ is rather small in the time period in which $d c_{\mathrm{K}}^{i} / d t$ varies appreciably.

A likely explanation for the apparent decrease of $v$ is the heterogeneity of the vesicle population. Since the formation of vesicles and the incorporation of ATPase molecules are random processes, both the vesicle diameter as well as the number of pump molecules per vesicle necessarily vary among the vesicle population. This means that the quantity $n_{P} / V$, the number of pumps per unit of internal volume, which determines $d c_{\mathrm{K}}^{i} / d t$ [Eq. (3)], is distributed over a certain range. Vesicles with a large value of $n_{P} / V$ lose their internal $\mathrm{K}^{+}$faster than vesicles with small $n_{P} / V$; thus, the number of active vesicles decreases in the course of the experiment.

As a consequence of the heterogeneity of the vesicle population, Eq. (3) can be applied only in an approximate sense for the determination of $v_{0}$. For a more accurate evaluation of the transport rate a statistical model of the vesicle population has been used which is described in detail in Appendix A and which is based on the assumption that the vesicle radii are normally distributed and that the number of pumps per vesicle is given by a Poisson distribution. For the mean vesicle radius $\bar{r}$ and the variance $\sigma^{2}=\overline{(r-\bar{r})^{2}}$ the values $\bar{r}=45 \mathrm{~nm}$ and $\sigma=5 \mathrm{~nm}$ were used, as obtained from the light-scattering experiments.

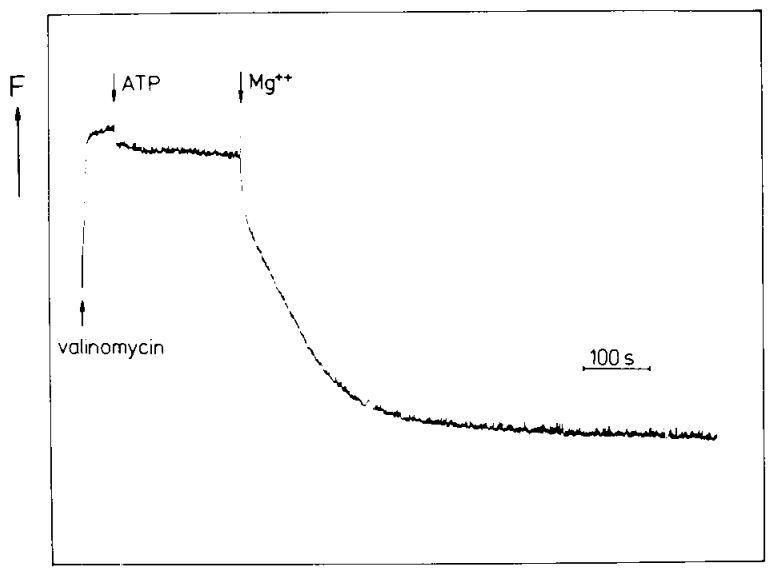

Fig. 5. Control experiment in which ATP was initially added in the absence of $\mathrm{Mg}^{2+}$. The small fluorescence decrease results from the dilution of the dye. After addition of $5 \mathrm{mM} \mathrm{Mg}^{2+}$ a large change of fluorescence intensity occurs. The other experimental conditions were the same as described in the legend to Fig. 4

The mean number $\bar{n}$ of pump molecules (ATPbinding site facing outward) per vesicle of radius $r$ was calculated according to $\bar{n}=4 \pi r^{2} \chi$ where $\chi$ is the constant density of outward-oriented pumps per unit area of the vesicle membrane. An upper limit of $\chi$ is given by the protein/lipid ratio of the vesicle preparation, assuming an efficiency of $100 \%$ of the reconstitution and a uniform (outward) orientation of the pump molecules. With a protein/lipid ratio (wt/wt) of 0.06 and a molar mass of $M=280,000$ $\mathrm{g} / \mathrm{mol}$ of Na,K-ATPase (Jørgensen, 1982), the upper limit of $\chi\left[\mathrm{Eq}\right.$. (A13)] becomes $640 \mu \mathrm{m}^{-2}$, corresponding to $\bar{n}=16$ for a vesicle of $r=45 \mathrm{~nm}$. The actual value of $\chi$ may be much smaller, however, since the efficiency of incorporation may be far less than $100 \%$ and only a fraction of the pump molecules may be oriented outward (Cornelius \& Skou, 1984). For this reason $\chi$ was used as an adjustable parameter in the fitting procedure. An optimum fit of Eq. (A8) to the experimentally observed time course of the fluorescence signal was obtained with $\chi$ values ion the range of $100-160 \mu \mathrm{m}^{-2}$, corresponding to values of $\bar{n}$ between 2.6 and 4.1 for $r=$ $45 \mathrm{~nm}$. The value of $\chi$ which gave an optimum fit was found to be rather insensitive to variations of the variance $\sigma^{2}$; increasing from 5 to $10 \mathrm{~nm}$ required an increase of $\chi$ by only 10 to $20 \%$.

The analysis, according to Appendix A, of the experiment represented in Fig. 4 yields (with $\chi=$ $160 \mu \mathrm{m}^{-2}$ ) a maximum transport rate $v_{m}$ of $12.3 \mathrm{~s}^{-1}$ at $16^{\circ} \mathrm{C}$. Nine independent experiments at $16^{\circ} \mathrm{C}$ with different vesicle preparations gave a mean value of $v_{m}=(13.2 \pm 0.9) \mathrm{s}^{-1}$. This value may be compared with the maximum turnover rate of the isolated kid- 


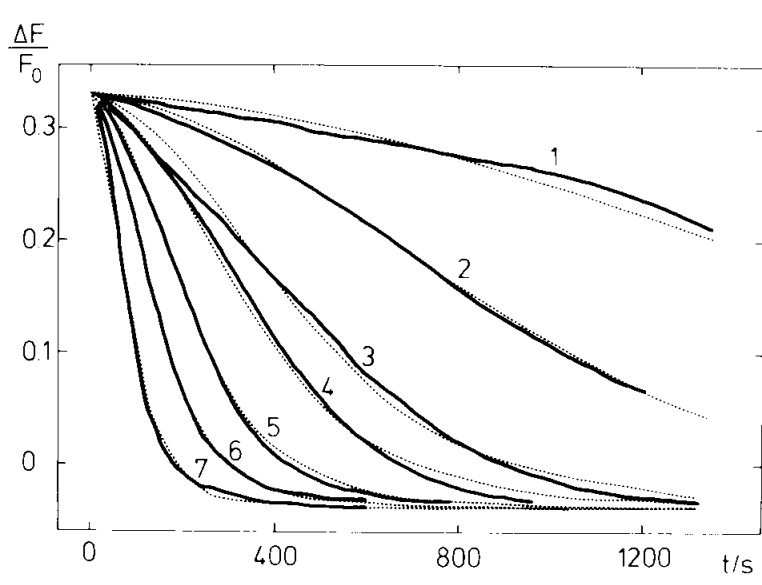

Fig. 6. Temperature dependence of ATP-driven extrusion of $\mathrm{K}^{-}$ Apart from temperature, the experimental conditions were the same as described in the legend to Fig. 4. (1) $6.4^{\circ} \mathrm{C}$; (2) $9.4^{\circ} \mathrm{C}$ : (3) $12.4^{\circ} \mathrm{C}$; (4) $15.3^{\circ} \mathrm{C}$; (5) $17.1^{\circ} \mathrm{C}$; (6) $19.7^{\circ} \mathrm{C}$; (7) $22.7^{\circ} \mathrm{C}$. The dotted lines are theoretical curves calculated from Eq. (A8) using the following fixed parameter values: $\bar{r}=45 \mathrm{~nm}, \sigma=5 \mathrm{~nm}, \Delta \mathrm{r}=2.5$ $\mathrm{nm}, x=120 \mu \mathrm{m}$ ?. The only adjustable parameter was the maximum transport rate $v_{m}$ [Eq. (A 10)] for which the following values were obtained from the fit procedure: (I) $1.9 \mathrm{~s}^{-1}$; (2) $4.0 \mathrm{~s}^{-1}$; (3)

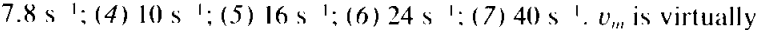
identical with the initial transport rate $v_{\text {s. }}$

ney enzyme before reconstitution which is of the order of $\sim 150 \mathrm{~s}^{-1}$ at $37^{\circ} \mathrm{C}$, and with previously determined turnover rates of $\mathrm{Na}, \mathrm{K}$-ATPase incorporated into artificial vesicles which are in the range of $1-50 \mathrm{~s}^{-1}$ (based on $\mathrm{Na}^{+}$-transport rates) at $22-25^{\circ} \mathrm{C}$ (Anner, 1980; Cornelius \& Skou, 1984). Determinations of the transport rate at variable temperature (see below) yield $v \simeq 40 \mathrm{~s}^{-1}$ at $23^{\circ} \mathrm{C}$; the extrapolated value of $v_{m}$ at $37^{\circ} \mathrm{C} \sim 300 \mathrm{~s}^{-1}$. Since the temperature range of our experiments was $6-23^{\circ} \mathrm{C}$, the extrapolation to $37^{\circ} \mathrm{C}$ is uncertain. Furthermore, the absolute values of $v_{m}$ are subjected to errors in the determination of pump density $\chi$.

While the absolute value of $v_{m}$ has to be evaluated by a fit of Eq. (A8) to $\Delta F / F_{o}$, a simpler procedure may be used for the determination of relative values of the pump rate. Simulations of $y(t)=\Delta F / F_{o}$ according to Eq. (8) show that the time $t_{c}$, required for $y$ to go through zero is proportional to $1 / v_{m}$ at fixed values of $\chi, \bar{r}$ and $\sigma$. Ratios of $t_{0}$, values may therefore be used in order to determine ratios of pump rates.

Another approximate method of determining pump rates consists in measuring $d y / d t$ at time $t=$ 0 . According to Eq. $(\mathrm{A} 14),(d y / d t)_{t=0}$ is proportional to the initial value $v_{0} \approx v_{m}$ of the pump rate if the vesicles are of uniform size. In case of a finite but narrow size distribution Eq. (A14) may be expected to be applicable still as an approximation. The mag-

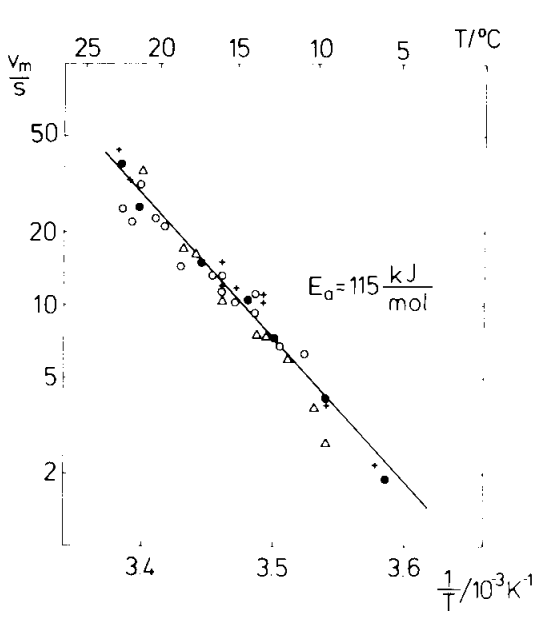

Fig. 7. Evaluation of the activation energy $E_{i}$, using data from Fig. 6(O) together with the results of three further sets of experiments with two other vesicle preparations $(O, \triangle$ and +$) . v_{m}$ is the maximum transport rate. The straight line has been drawn according to Eq. (4) with $E_{a}=115 \mathrm{~kJ} / \mathrm{mol}$

nitude of the error introduced by using Eq. (A14) instead of Eq. (A8) may be estimated by comparing the pump rates per vesicle determined by the two methods from the data of Fig. 4. Using Eq. (A14), $\bar{n} v_{0}$ is determined to be $80 \mathrm{~s}^{-1}$, whereas the fit procedure according to Eq. (A8) yields $\bar{n} v_{m} \simeq \bar{n} v_{0}=50$ $\mathrm{s}^{-1}$.

\section{Temperature Dependence OF TRANSPORT RATES}

The time course of the fluorescence signal after ATP addition strongly depends on temperature. In Fig. $6 \Delta F / F_{\text {o }}$ as a function of time $t$ is plotted for seven different temperatures between 6.4 and $22.7^{\circ} \mathrm{C}$. The transport rates $v_{m}$ have been determined by the fit procedure described in the previous section using the following parameter values: $\bar{r}=45$ $\mathrm{nm}, \sigma=5 \mathrm{~nm}$, and $\chi=120 \mu \mathrm{m}^{-2}$. The only adjustable parameter in the calculated fit curves (dotted lines in Fig. 6) was the transport rate $v_{m}$. The fact that the shape of the $\Delta F / F_{\text {o }}$ curves in the whole temperature range could be satisfactorily fitted by varying a single parameter $\left(v_{m}\right)$ supports the assumptions introduced in the derivation of Eq. (A8).

For the determination of the activation energy $E_{a}$, values of $v_{m}$ obtained at different temperatures $T$ are plotted in Fig. 7 on a logarithmic scale as a function of $1 / T$, according to the Arrhenius equation:

$v_{m}=v_{m}^{\prime \prime} \exp \left(-E_{a} / R T\right)$ 


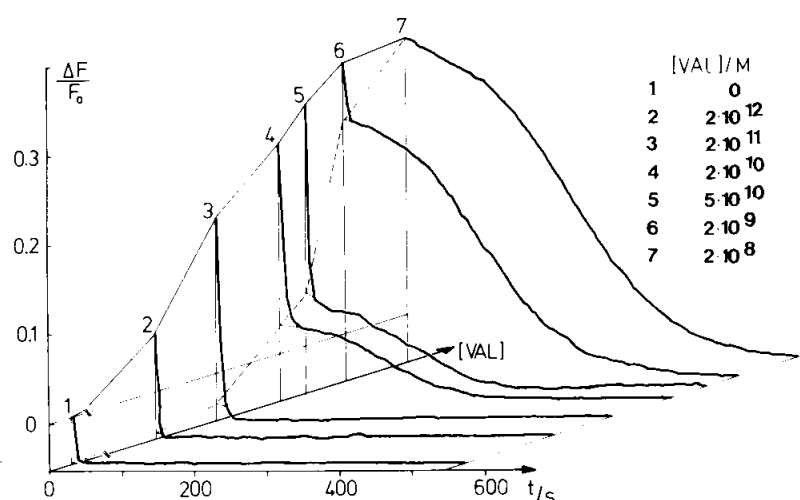

Fig. 8. Time course of the fluorescence signal for different valinomycin concentrations. The signal starts after ATP addition at time $t=0$. For $t<0$ the signal is determined by a diffusion potential which approaches the Nernst potential for potassium $\left(c_{\mathrm{K}}^{\mathrm{i}} / \mathrm{c}_{\mathrm{K}}^{\prime}=14\right)$ at high valinomycin concentration. The fast change

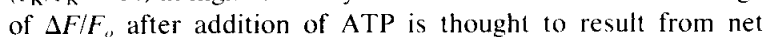
charge transport by the pump leading to a voltage drop across the membrane resistance. The experimental conditions (apart from the variable valinomycin concentration) were the same as indicated in the legend of Fig. 4

$v_{m}^{o}$ is a temperature-independent constant. From the slope of the straight line in Fig. 7 an activation energy of $E_{t}=115 \mathrm{~kJ} / \mathrm{mol}$ ( $\left.=27 \mathrm{kcal} / \mathrm{mol}\right)$ is obtained; corresponding to $\mathrm{Q}_{10}=5.3$. The value of $E_{a}$ may be compared with the activation energy $E_{a}=71 \mathrm{~kJ} / \mathrm{mol}$ of ATP hydrolysis of the Na,K-ATPase from guinea-pig kidney (Post, Sen \& Rosenthal, 1965). A more direct comparison is possible with tracer-flux studies of active $\mathrm{Na}^{+}$transport in reconstituted egg lecithin vesicles containing $\mathrm{Na}, \mathrm{K}$-ATPase from $\mathrm{rab}-$ bit kidney (Anner \& Moosmayer, 1982); the temperature dependence of the observed flux rates corresponds to an activation energy of $80 \mathrm{~kJ} / \mathrm{mol}$. The reason for the difference in the $E_{a}$ values of ion transport $(80$ and $115 \mathrm{~kJ} / \mathrm{mol}$ ) is not clear so far; it possibly results from the difference in the lipids used for reconstitution (egg lecithin and dioleoyllecithin).

\section{Electrogenic Effect of the Pump}

When the valinomycin concentration is reduced, the fluorescence signal changes its shape. After addition of ATP the fluorescence intensity quickly falls to a lower level and thereafter declines more slowly (Fig. 8). The time course of the fast signal change is limited by the mixing time of the solution in the fluorescence cell which is about $3 \mathrm{sec}$. The drop of $\Delta F$ is thought to reflect a fast change of membrane voltage resulting from the electrogenic action of the pump. The direction of the signal

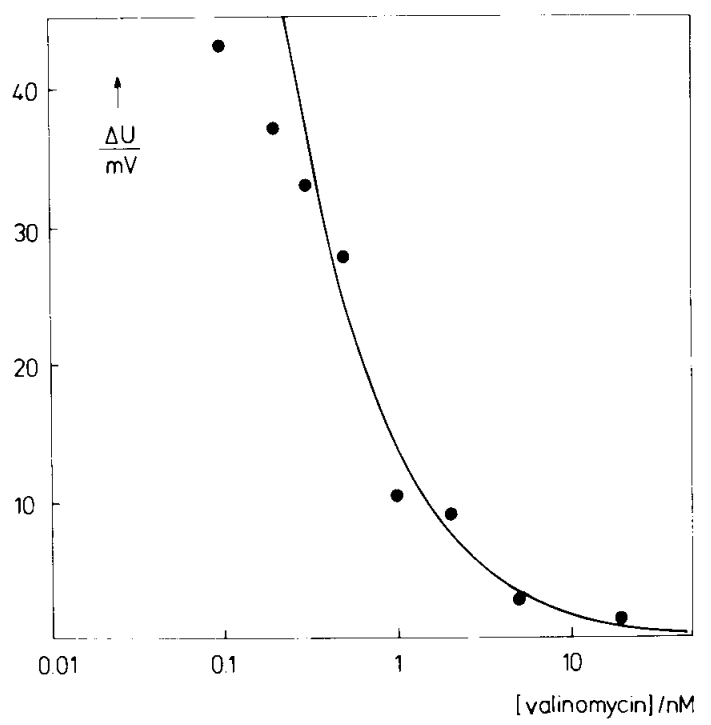

Fig. 9. Amplitude $\Delta U=U^{*}-U_{n}$ of the fast voltage change after ATP addition as a function of valinomycin concentration. $\Delta U$ represents the voltage drop created by the pump current across the membrane resistance. $U_{v}$ is the diffusion potential prior to the addition of valinomycin and $U^{*}$ the voltage at the end of the fast process. $U_{0}$ and $U^{*}$ have been obtained from the $\Delta F / F_{0}$ values given in Fig. 8 using the calibration curve from Fig. 2. Data from experiments with valinomycin concentrations below $0.1 \mathrm{~nm}$ have not been plotted because of the uncertainty in the conversion of $\Delta F / F_{0}$ to $U$ at positive values of $U$ (Fig. 2). The line has been drawn according to Eqs. (18) and (19) (with $U_{0} \equiv$ $\left.R T u_{u} / F, U^{*} \equiv R T_{u}^{*} / F\right)$ using the following parameter values: $\alpha$ $=5.2 \times 10^{-13} \mathrm{M}^{2},(\nu-\kappa) n_{\nu} v_{0}=80 \mathrm{~s}^{-1}, P_{\mathrm{K}}=\left(4.4 \mathrm{~cm} \mathrm{M}^{-1} \mathrm{~s}{ }^{1}\right)[\mathrm{val}]$

change is consistent with the assumption that the pump translocates more $\mathrm{Na}^{+}$ions inward than $\mathrm{K}^{+}$ ions outward, thus shifting the electrical potential of the vesicle interior towards positive values.

It is seen from Fig. 8 that the amplitude of the fast signal change goes through a maximum at intermediate valinomycin concentrations and decreases again as the valinomycin concentration is reduced to zero. This decline is consistent with the observation (Fig. 3) that at low valinomycin concentration the membrane voltage is already close to zero (much smaller than the Nernst potential) prior to the addition of ATP; when the pump is activated, the potential inside the vesicle rises to positive values at which the fluorescence signal saturates (Fig. 2).

The amplitude $U^{*}-U_{0}$ of the fast voltage change is plotted in Fig. 9 as a function of valinomycin concentration. $U_{0}$ is the voltage prior to the addition of valinomycin and $U^{*}$ the voltage at the end of the fast process. The difference $\Delta U \equiv U^{*}-U_{0}$ represents the voltage drop created by the pump current across the membrane resistance. It is seen 
from Fig. 9 that, in accordance with expectation, $\Delta U$ strongly increases with decreasing valinomycin concentration, i.e., increasing membrane resistance.

The experimental results represented in Fig. 8 are consistent with the assumption that the reconstituted $\mathrm{Na}, \mathrm{K}$ pump from kidney transports net charge across the vesicle membrane. The electrogenicity of the $\mathrm{Na}, \mathrm{K}$ pump is well documented from experiments with erythrocytes (Hoffman, Kaplan \& Callahan, 1979), with squid giant axons (Abercrombie \& De Weer, 1978), heart cells (Glitsch, 1982), and barnacle muscle fibers (Lederer \& Nelson, 1984). In reconstituted systems the electrogenic nature of $\mathrm{Na}^{+}, \mathrm{K}^{+}$transport has been inferred from experiments using lipophilic ions such as $\mathrm{SCN}^{-}$or triphenylmethylphosphonium (Dixon \& Hokin, 1980; Forgac \& Chin, 1982).

\section{Discussion}

Optical Measurement of Membrane Voltage AND $\mathrm{K}^{+}$CONCENTRATION

The fluorescence method described allows the performance of fast and continuous measurements of ATP-driven potassium fluxes in membrane vesicles. Compared to tracer-flux experiments, the optical technique yields a more accurate determination of flux rates. This is particularly important since the ion content of small vesicles significantly changes within seconds after activation of the $\mathrm{Na}, \mathrm{K}$-pump. In the present study in which a conventional mixing device has been used, the increased time-resolution of the optical method has not been fully exploited, however.

The use of optical probes for ion-flux studies is limited to some extent by the fact that the mechanism by which a dye responds to changes of membrane voltage is not yet fully understood (Sims, Waggoner, Wang \& Hoffman, 1974). Since the absorption and emission spectrum of organic dyes depends on solvent polarity, a possible mechanism for the voltage-sensitivity consists in a field-induced shift of the position of the dye in the membranesolution interface and a concomitant change of polarity in the immediate vicinity of the dye molecule (Waggoner, 1979). Consistent with this interpretation is the finding that adsorption of $1,3,3,1^{\prime}, 3^{\prime}, 3^{\prime}-$ hexamethylindodicarbocyanine (NK 529) from the aqueous medium to lipid vesicles leads to a red shift of the emission spectrum, whereas creation of an inside-positive potential (which tends to move the cationic dye towards the aqueous side of the interface) is accompanied by a blue shift (Lüdi et al., 1981). It is likely, however, that more than one process is responsible for the voltage sensitivity of NK529; Ross et al. (1977) observed a biphasic fluorescence signal from nerve axons stained with NK529 after application of a voltage step, consisting of a fast component with a time constant below $100 \mu \mathrm{sec}$ and a second slower component with a time constant of about $10 \mathrm{msec}$. The slower signal may result from a change in the monomer/dimer equilibrium of the membrane-bound dye (Ross et al., 1977).

While the dye is likely to be primarily a polarity sensor, it may be used as a voltage indicator if the optical signal is a unique function of voltage under the given experimental conditions. In the experiments summarized in Fig. 2 it has indeed been found (in a certain range of $c_{\mathrm{K}}^{e}$ and $c_{\mathrm{K}}^{i}$ ) that the fluorescence signal $\Delta F / F_{o}$ depends only on the concentration ratio $c_{\mathrm{K}}^{e} / c_{\mathrm{K}}^{i}$ determining the Nernst potential, irrespective of the absolute values of $c_{\mathrm{K}}^{i}$ and $c_{\mathrm{K}}^{e}$. Furthermore, the relationship between $\Delta F / F_{o}$ and the voltage was found to be virtually identical for pure lipid vesicles and vesicles containing $\mathrm{Na}, \mathrm{K}$ ATPase. This test for the absence of protein effects on $\Delta F / F_{o}$ is important, since experiments of Lüdi et al. (1983) with acetylcholine-receptor vesicles and isolated SR of skeletal muscle (Oetliker, 1980, 1981), have shown that the indocyanine dye may interact with membrane proteins. For discussion of potential independent signals see Beeler, Farmen and Martonosi (1981) and Oetliker (1982).

When dye adsorbs to the vesicle, the interfacial potential of the vesicle membrane may be changed (Krasne, 1983). If at a total dye concentration of 2.3 $\mu \mathrm{M}$ and a lipid concentration of $0.1 \mathrm{mg} / \mathrm{ml}(130 \mu \mathrm{M})$ all dye molecules added to the vesicle suspension bind to the vesicle surface, the molar ratio lipid/dye is $60: 1$ and the interfacial charge density $6 \times 10^{4}$ $e_{0} / \mu \mathrm{m}^{2}\left(e_{0}\right.$ is the elementary charge). At an ionic strength of $0.2 \mathrm{M}$ the interfacial potential is then calculated from the Gouy-Chapman relation (McLaughlin, 1977) to be $9 \mathrm{mV}$. This means that effects of net charge of adsorbed dye molecules are rather small. It cannot be excluded, however, that dye adsorption modifies the dipolar potential of the membrane. An asymmetric change of dipolar potential would alter the electrical field strength in the membrane and thus could affect the pump rate. Such an effect of dye adsorption is unlikely, however, since the time course of the fluorescence signal after ATP addition was found to be insensitive against a tenfold reduction of dye concentration.

In the measurement of $\mathrm{K}^{+}$fluxes in the pres- 
ence of valinomycin the vesicle membrane acts as a $\mathrm{K}^{+}$-specific electrode; variations in the $\mathrm{K}^{+}$content of the vesicle are detected as concentration changes. This means that the method is subjected to possible artifacts from changes in the vesicle volume resulting from osmotic effects. In the potassium flux experiments described above, osmotic effects are negligible, however, since (as discussed previously) the potassium efflux is always equal to the sodium influx in the presence of a high valinomycin concentration.

The sensitivity of the potassium flux measurement in the presence of valinomycin can be estimated from the minimum voltage change $\Delta U$ detectable in the fluorescence record, which is about 3 $\mathrm{mV}$. According to Eq. (1) this yields for the sensitivity of the concentration measurement:

$\frac{\Delta c_{\mathrm{K}}^{i}}{c_{\mathrm{K}}^{i}}=\frac{\Delta U}{R T / F}=0.1$.

This means that a $10 \%$ change of internal $\mathrm{K}^{+}$concentration is detectable. A spherical vesicle with 40 $\mathrm{nm}$ internal radius contains about $16,000 \mathrm{~K}^{+}$ions at $c_{\mathrm{K}}^{i}=0.1 \mathrm{M} ;$ a $10 \%$ variation of $c_{\mathrm{K}}^{i}$ thus corresponds to $\sim 500$ pump turnovers.

A much higher sensitivity is given at low membrane conductance (in the absence of ionophore). In this case the net charge translocated by the pump is directly related to the voltage $\Delta U$ across the membrane capacitance. If $A$ is the surface area of the vesicle and $C_{m} \simeq 1 \mu \mathrm{F} / \mathrm{cm}^{2}$ the specific membrane capacitance, the number $\Delta n$ of univalent ions needed to build up a voltage $\Delta U=3 \mathrm{mV}$ across the membrane is

$\Delta n=\left(C_{m} / e_{o}\right) A \Delta U \simeq 4$

( $e_{0}=1.6 \times 10^{-19} \mathrm{C}$ is the elementary charge). Thus, a few pump turnovers can easily be detected if the resistance of the membrane is high.

\section{Time Dependence of Membrane Voltage}

In the following we give a more general analysis of the experimental results which describes the time course of membrane voltage $U$ as a function of passive $\mathrm{K}^{+}$permeability. At any time $t$ during the flux experiment, the rate of change of $U$ is equal to the net electric current $l$ through the membrane, divided by the membrane capacitance $A C_{m}$ :

$$
-A C_{m} \frac{d U}{d t}=I=e_{o}\left(J_{\mathrm{K}, a}+J_{\mathrm{K}, p}+J_{N, a}\right)+A \lambda U
$$

The second part of Eq. (7) is based on the assumption that the total current $I$ can be divided into contributions from active and passive sodium and potassium fluxes and from passive leakage of other ions. $J_{\mathrm{K}, a}$ and $J_{\dot{N}_{. a}}$ are the active fluxes $\left(\mathrm{s}^{-1}\right)$ of $\mathrm{K}^{+}$ and $\mathrm{Na}^{+}$, respectively, per vesicle, and $J_{\mathrm{K} . p}$ is the passive $\mathrm{K}^{+}$flux. Outward fluxes are counted positive and inward fluxes negative. The passive $\mathrm{Na}^{+}$ flux which is likely to be small under the conditions of our experiments has been combined with the residual leakage flows which are described by a specific conductance $\lambda$. In Eq. (7) and in the following equations, effects of vesicle heterogeneity are not taken explicitly into account. Quantities such as $A$ or $n_{P}$ thus have to be considered as averages.

The active ion fluxes $J_{\mathrm{K} . a}$ and $J_{N . a}$ are proportional to the product $n_{P} v$ [compare Eq. (3)]:

$J_{\mathrm{K}, a}=\kappa n_{P} v ; J_{\mathrm{N}, \mathrm{a}}=-\nu n_{P} v$.

$\kappa$ and $\nu$ denote the number of $\mathrm{K}^{+}$and $\mathrm{Na}^{+}$ions, respectively, which are translocated per turnover. The passive $\mathrm{K}^{+}$flux, which is a function of the internal and external $\mathrm{K}^{+}$concentrations as well as of voltage, is given by the Goldman equation (Goldman, 1943):

$J_{\mathrm{K}, \mathrm{p}}=A P_{\mathrm{K}} u \frac{c_{\mathrm{K}}^{i} \exp (u)-c_{\mathrm{K}}^{e}}{\exp (u)-1}$

$u \equiv U F / R T$.

$u$ is the membrane voltage in units of $R T / F=25$ $\mathrm{mV}$. The permeability coefficient $P_{\mathrm{K}}$ may depend on voltage and ion concentration in the case of carriermediated ion transport (Läuger \& Stark, 1970); this complication is omitted in the following. Introduction of Eqs. (8)-(10) into Eq. (7) yields for the rate of change of voltage $u$ :

$\frac{d u}{d t}=\frac{e_{u} F}{R T A C_{m}}\left[(\nu-\kappa) n_{P} v-A P_{\mathrm{K}} u \frac{C_{\mathrm{K}}^{i} \exp (u)-c_{\mathrm{K}}^{e}}{\exp (u)-1}\right]-\frac{\lambda u}{C_{m}} \cdot$

The value of the dimensionless quantity $e_{0} F / R T A C_{\mathrm{m}}$ is about 0.03 for a spherical vesicle of radius $r_{e}=45$ $\mathrm{nm}$. As discussed above, the external $\mathrm{K}^{+}$concentration $c_{\mathrm{K}}^{e}$ remains virtually constant in the flux experiments. The internal $\mathrm{K}^{+}$concentration decreases with time according to $d c_{\mathrm{K}}^{i} / d t=-\left(J_{\mathrm{K}, a}+J_{\mathrm{K}, p}\right) / V$ :

$-V \frac{d c_{\mathrm{K}}^{i}}{d t}=\kappa n_{P} v+A P_{\mathrm{K}} u \frac{c_{\mathrm{K}}^{i} \exp (u)-c_{\mathrm{K}}^{e}}{\exp (u)-1}$.

Eqs. (11) and (12) represent two coupled differential equations for the functions $u(t)$ and $c_{\mathrm{K}}^{i}(t)$. The discussion of Eqs. (11) and (12) may be considera- 
bly simplified, since the time course of membrane voltage $u$ is governed by two widely different time constants. After activation of the pump, the membrane capacitance is charged up to a quasistationary voltage which is determined by the pump rate and by the total membrane conductance $\lambda_{t}$. The time constant for this charging process is

$\tau_{c h}=C_{m} / \lambda_{t}$

$\lambda_{t}$ is the sum of the leakage conductance $\lambda$ introduced above and of the valinomycin-induced potassium conductance. A lower limit of $\lambda_{t}$ may be estimated from the passive ionic permeability of Na,K-ATPase vesicles (Anner, 1981) which yields $\lambda_{t} \geq 100 \mathrm{nS} / \mathrm{cm}^{2}$. Together with $C_{m} \simeq 1 \mu \mathrm{F} / \mathrm{cm}^{2}$, the charging time is estimated to be $\tau_{\mathrm{ch}} \leqslant 10 \mathrm{sec}$. In the presence of valinomycin, $\tau_{\text {ch }}$ may become much smaller than $10 \mathrm{sec}$. The initial rate of voltage change depends only on the pump rate and the membrane capacitance:

$\left(\frac{d U}{d t}\right)_{i-0}=(\nu-\kappa) \frac{n_{P} v_{\omega} e_{o}}{A C_{m}}$

$v_{0}$ is the initial turnover rate. With $(\nu-\kappa) n_{P} v_{0} \simeq 80$ $\mathrm{s}^{-1}$ (see above) $d U / d t$ becomes $\sim 60 \mathrm{mV} / \mathrm{sec}$.

After the fast charging-up process, the time course of $u$ is dominated by a slow change of $\mathrm{K}^{+}$ diffusion potential resulting from the decrease of internal $\mathrm{K}^{+}$concentration. This quasistationary process is virtually electroneutral, consisting (in the presence of valinomycin) in a $1: 1$ exchange of $\mathrm{K}^{+}$ for $\mathrm{Na}^{+}$. If $\tilde{c}_{\mathrm{K}}^{i}$ is the internal $\mathrm{K}^{+}$concentration at time $t<0$ (prior to the addition of ATP), the characteristic time of the exchange process is given by

$\tau_{\mathrm{ex}} \equiv\left[\left(\frac{1}{c_{\mathrm{K}}^{i}} \frac{d c_{\mathrm{K}}^{i}}{d t}\right)_{t=0}\right]^{-1}=\frac{\tilde{c}_{\mathrm{K}}^{i} V}{\nu n_{P} v_{t}}$

Under the experimental conditions of Fig. 4 the exchange time is estimated to be $\tau_{\mathrm{ex}} \simeq 130 \mathrm{sec}$, much larger than $\tau_{\text {ch }}$.

Since the slow process is determined by the conditions $I \approx 0$ and $c_{\mathrm{K}}^{i}=\tilde{c}_{\mathrm{K}}^{i}-\nu n_{p} v t / V$, one obtains instead of Eq. (11):

$(\nu-\kappa) \frac{n_{p} v}{A P_{\mathrm{K}}}=u \frac{\left(c_{\mathrm{K}}^{i}-\nu n_{p} v t / V\right) \exp (u)-c_{\mathrm{K}}^{\prime}}{\exp (u)-1}+q u$

$q \equiv R T \lambda / e_{0} F P_{K}$.

Eq. (16) represents an implicit relation for $u(t)$ in the time domain of the slow process.

Prior to the activation of the pump $(t<0)$, the membrane voltage represents a pure diffusion po- tential. Introducing the condition $v=0$ into Eq. (16), this initial voltage is obtained as

$u_{o}=\ln \frac{c_{\mathrm{K}}^{i}+q}{c_{\mathrm{K}}^{i}+q}=\ln \frac{c_{\mathrm{K}}^{i}+\alpha / c_{v}}{c_{\mathrm{K}}^{i}+\alpha / c_{v}}$

The second part of Eq. (18) holds under the assumption that the potassium permeability coefficient $P_{\mathrm{K}}$ is proportional to the valinomycin concentration $C_{k}$ ( $\alpha$ is a constant). As seen from Fig. 3, the experimentally observed dependence of the diffusion potential on valinomycin concentration is satisfactorily described by Eq. (18). With the value of $\alpha$ used for the fit of the experimental data of Fig. 3, $\alpha=5.2$ $\times 10^{-13} \mathrm{M}^{2}$, and with the estimated value of the $\mathrm{K}^{+}$ permeability coefficient at $c_{v}=10 \mathrm{nM}\left(P_{\mathrm{K}}=1 \times\right.$ $10^{-7} \mathrm{~cm} / \mathrm{sec} ; \mathrm{sec}$ below), the leakage conductance becomes $\lambda=20 \mathrm{nS} / \mathrm{cm}^{2}$.

When the pump is activated at time $t=0$, the membrane voltage rises within a short time $t \simeq \tau_{\mathrm{ch}}$ to the initial value $u^{*}$ for the slow process. According to Eq. (16), $u^{*}$ is given by

$(\nu-\kappa) \frac{n_{P} v_{u}}{A P_{\mathrm{K}}}=u^{*} \frac{\tilde{c}_{\mathrm{K}}^{i} \exp \left(u^{*}\right)-c_{\mathrm{K}}^{e}}{\exp \left(u^{*}\right)-1}+q u^{*}$.

As already mentioned, the difference $\Delta U=(R T / F)$ $\left(u^{*}-u_{0}\right)$ represents the voltage drop across the membrane resistance resulting from the electrogenic action of the pump. This is easily seen in the special case $c_{\mathrm{K}}^{i}=c_{\mathrm{K}}^{e} \equiv c_{\mathrm{K}}$ in which the membrane resistance is given by $1 / \mathrm{A}\left(\lambda+\lambda_{K}\right)$ where $\lambda_{K}=$ $c_{\mathrm{K}} P_{\mathrm{K}} e_{0} F / R T$ is the valinomycin-induced membrane conductance. Under this condition the relation $\Delta U$ $=I_{P} / A\left(\lambda+\lambda_{\mathrm{K}}\right)$ holds according to Eqs. (18) and (19), $I_{P}=(\nu-\kappa) e_{o} n_{P} v$ being the pump current.

For high potassium permeability $\left(P_{\mathrm{K}} \rightarrow \infty, q \approx\right.$ 0 ) Eqs. (18) and (19) predict $u^{*} \approx u_{0} \approx \ln \left(c_{\mathrm{K}}^{e} / \tilde{c}_{\mathrm{K}}^{i}\right)$, or $\Delta U=0$, in accordance with the experimental finding (Fig. 9). On the other hand, in the absence of valinomycin $\left(P_{\mathrm{K}} \approx 0, q \rightarrow \infty, u_{0} \approx 0\right)$ the relation $\Delta U$ $\approx I_{P} / A \lambda$ holds. With $(\nu-\kappa) n_{P} v_{0}=80 \mathrm{~s}^{-1}$ (see above), the pump current density $I_{p} / A$ becomes $\sim 60$ $\mathrm{nA} / \mathrm{cm}^{2}$; using the previously estimated value of the leakage conductance, $\lambda \simeq 30 \mathrm{nS} / \mathrm{cm}^{2}, U$ is calculated to be $\sim 2 \mathrm{~V}$, which is far above the thermodynamic reversal potential $U_{r}$ of the pump. When $U$ approaches $U_{r}$, the (voltage-dependent) pump rate slows down so that $U$ never exceeds $U_{r}$. Nevertheless, the estimate given above indicates that for plausible values of the leakage conductance $\lambda$, the membrane voltage may come close to the reversal potential of the pump.

An experimental test of Eq. (19) is represented in Fig. 9. Values of $P_{\mathrm{K}}$ may be estimated from mea- 
surements of valinomycin-induced potassium conductance $\lambda_{K}$ in artificial planar bilayer membranes made from dioleoylphosphatidylcholine, using the relation $\lambda_{\mathrm{K}}=c_{\mathrm{K}} P_{\mathrm{K}} e_{0} F / R T$. From the experiments of Stark and Benz (1971) the empiric relation $P_{\mathrm{K}} \simeq$ $\left(22 \mathrm{~cm} \mathrm{M}^{-1} \mathrm{~s}^{-1}\right) c_{v}^{a}$ is obtained where $c_{v}^{a}$ is the aqueous valinomycin concentration. According to Eq. (2), $c_{v}^{a}$ is related to the total valinomycin concentration $c_{v} \equiv[\mathrm{val}]$ in the vesicle suspension by $c_{v}^{a}=(1-$ $\theta) c_{v}=0.2 c_{v}$. The best fit of Eqs. (18) and (19) to the experimental points is obtained with the relation $P_{\mathrm{K}}$ $=\left(50 \mathrm{~cm} \mathrm{M}^{-1} \mathrm{~s}^{-1}\right) c_{v}^{a}$, when the previously estimated parameters $(\nu-\kappa) n_{p} v_{o}=80 \mathrm{~s}^{-1}$ and $\alpha=7 \times 10^{-13}$ $\mathrm{M}^{2}$ are used. It is seen from Fig. 9 that the theoretical curve adequately describes the observed dependence of $\Delta U$ on valinomycin concentration.

The authors wish to thank Mrs. Roudna and Mrs. Moosmayer for excellent technical assistance, Dr. H. Alpes for lipid analysis. and Prof. H.G. Weder and Dr. R.A. Schwendener for the dynamic light-scattering experiments. We wish to thank Dr. M.T. Nelson for valuable comments on the manuscript.

This work has been financially supported by Deutsche Forschungsgemeinschaft (Sonderforschungsbereich 156) and the Swiss National Science Foundation grants Nr. 3.536-0.83 to B.A. and $\mathrm{Nr}$. 3.322-0.82 to $\mathrm{H}$. (Oe.

\section{References}

Abercrombie, R., De Weer, P. 1978. Electric current generated by squid axon: External $\mathrm{K}$ and internal ADP effects. $A m . J$. Physiol. 44:389-400

Abeywardena, M.Y.. Allen. Th.M., Charnock, J.S. 1983. Lipidprotein interactions of reconstituted membrane-associated adenosine triphosphatases. Use of a gel-filtration procedure to examine phospholipid-activity relationships. Biochim. Biophys. Acta 729:62-74

Anner, B.M. 1980. Ratio of $\mathrm{Na}: \mathrm{K}$ tranpsort in reconstituted sodium pump vesicles. Biochem. Biophys. Res. Commun. 94:1233-1241

Anner, B.M. 1981. A K-selective cation channel formed by $\mathrm{Na}, \mathrm{K}$-ATPase in liposomes. Biochem. Int. 2:365-371

Anner, B.M., Lane, L.K., Schwartz, A., Pitts, B.J.R. 1977. A reconstituted $\mathrm{Na}^{+}+\mathrm{K}^{+}$pump in liposomes containing purified $\left(\mathrm{Na}^{+}+\mathrm{K}^{+}\right)$-ATPase from kidney medulla. Biochim. Biophys. Acta 467:340-345

Anner, B.M., Marcus, M.M., Moosmayer, M. 1984a. Reconstitution of Na,K-ATPase. In: Enzymes, Receptors and Carriers of Biomembranes. pp. 81-96. Springer-Verlag. Heidelberg

Anner, B.M., Moosmayer, M. 1981. Preparation of Na.KATPase-containing liposomes with predictable transport properties by a procedure relating the Na.K-transport capacity to the ATPase activity. J. Bichem. Biophys. Methods 5:299-306

Anner, B.M., Moosmayer, M. 1982. On the kinetics of the Na: K exchange in the initial and final phase of sodium pump activity in liposomes. J. Membr. Sci. 11:27-37

Anner, B.M., Robertson, J.D., Ting-Beall, H.P. 1984b. Characterization of $\left(\mathrm{Na}^{+}+\mathrm{K}^{+}\right)$-ATPase liposomes. I. Effect of enzyme concentration and modification of liposome size, intramembrane particle formation and $\mathrm{Na}^{+}, \mathrm{K}^{+}$-transport. Biochim. Biophys. Acta 773:253-261
Bartlett, G. 1959. Phosphorous assay in column chromatography. J. Biol. Chem. 234:466-468

Beeler, T.J., Farmen, R.H.. Martonosi, A.N. 1981. The mechanism of voltage-sensitive dye responses on sarcoplasmic re ticulum. J. Membrane Biol. 62:113-137

Benz, R., Stark, G., Janko, K., Läuger, P. 1973. Valinomycinmediated ion transport through neutral lipid membranes: Influence of carbon chain length and temperature. J. Membrane Biol. 14:339-364

Brotherus, J.R., Jacobsen, L.. Jørgensen, P.L. 1983. Soluble and enzymatically stable $\left(\mathrm{Na}^{+}+\mathrm{K}^{+}\right)$-ATPase from mammalian kidney consisting predominantly of protomer $\alpha \beta$-units. Preparation, assay and reconstitution of active $\mathrm{Na}^{+}, \mathrm{K}^{+}$transport. Biochim. Biophys. Acta 731:290-303

Cantley, L.C. 1981. Structure and mechanism of the (Na,K)ATPase. Curr. Top. Bioenerg. 11:201-237

Carruthers, A., Melchior, D.L. 1983. Studies of the relationship between bilayer water permeability and bilayer physical state. Biochemistry 22:5797-5807

Cornelius, F., Skou. J.C. 1984. Reconstitution of $\left(\mathrm{Na}^{+}+\mathrm{K}^{+}\right)$ATPase into phospholipid vesicles with full recovery of its specific activity. Biochim. Biophys. Acta 772:357-373

Dixon, J.F.. Hokin. L.E. 1980). The reconstituted (Na,K)ATPase is electrogenic. J. Biol. Chem. 255:10681-10686

Forbush, B., III, 1984. An apparatus for rapid kinetic analysis of isotopic efflux from membrane vesicles and of ligand dissociation from membrane proteins. Anal. Biochem. (in press)

Forgac, M., Chin, G. 1982. $\mathrm{Na}^{-}$transport by the $\left(\mathrm{Na}^{+}\right)$-stimulated adenosine triphosphatase. J. Biol. Chem. 257:56525655

Glitsch, H.G. 1982. Electrogenic Na pumping in the heart. Annu. Rev. Physiol. 44:389-400

Goldin, S.M., Tong, S.W. 1974. Reconstitution of active transport catalyzed by the purified sodium and potassium ion-stimulated adenosine triphosphatase from canine renal medulla. J. Biol. Chem. 249:5907-5915

Goldman, D.E. 1943. Potential, impedance, and rectification in membranes. J. Gen. Physiol. 27:37-60

Hilden, S., Rhee, H.M., Hokin, L.E. 1974. Sodium transport by phospholipid vesicles containing purified sodium and potassium ion-activated adenosine triphosphatase. J. Biol. Chem. 249:7432-7440

Hoffman, J.F., Kaplan, J.H., Callahan, T.J. 1979. The Na: K pump in red cells is electrogenic. Fed. Proc. 38:2440-2441

Hoffman, J.F.. Laris, P.C. 1974. Determination of membrane potentials in human and Amphiuma red blood cells by means of a fluorescent probe. J. Physiol. (London) 239:519-552

Jackson, R.L., Verkleij, A.J., Zoelen, E.J.J. van, Lane. L.K., Schwartz, A., Deenen, L.L.M. van 1980. Asymmetric incorporation of $\mathrm{Na}^{+}, \mathrm{K}^{+}$-ATPase into phospholipid vesicles. Arch. Biochem. Biophys. 200:269-278

Jørgensen, P.L. 1974. Isolation of $\left(\mathrm{Na}^{+}+\mathrm{K}^{+}\right)$-ATPase. Methods Enzymol. 32:277-290

Jørgensen, P.L. 1982. Mechanism of the $\mathrm{Na}^{+}, \mathrm{K}^{+}$pump. Protein structure and conformations of the pure $\left(\mathrm{Na}^{+}+\mathrm{K}^{+}\right)$-ATPase. Biochim. Biophys. Acta 694:27-68

Karlish, S.J.D., Lieb, W.R., Stein, W.D. 1982. Combined effects of ATP and phosphate on rubidium exchange mediated by $\mathrm{Na}-\mathrm{K}-\mathrm{ATPase}$ reconstituted into phospholipid vesicles. $J$. Physiol. (London) 328:333-350

Karlish, S.J.D., Pick, U. 1981. Sidedness of the effects of sodium and potassium ions on the conformational state of the sodium-potassium pump. J. Physiol. (London) 312:505529 
Krasne, S. 1983. Interactions of voltage-sensing dyes with membranes. III. Electrical properties induced by merocyanine 540. Biophys. J. 44:305-314

Läuger, P., Stark, G. 1970. Kinetics of carrier-mediated ion transport across lipid bilayer membranes. Biochim. Biophys. Acta 211:458-466

Lawaczeck, R. 1979. On the permeability of water molecules across vesicular lipid bilayers. J. Membrane Biol. 51:229-261

Lederer, W.J., Nelson, M.T. 1984. Sodium pump stoichiometry determined by simultaneous measurements of sodium efflux and membrane current in barnacle. J. Physiol. (London) 348:665-677

Lowry, O.H., Rosebrough, N.J., Farr, A.L., Randall, R.J. 1951. Protein measurement with the Folin phenol reagents. J. Biol. Chem. 193:265-275

Lüdi, H., Oetliker, H., Brodbeck, U. 1981. Use of a potentiometric cyanine dye in the study of reconstituted membrane proteins. In: Membrane Proteins. A. Azzi, U. Brodbeck, and P. Zahler, editors. pp. 209-219. Springer, Berlin

Lüdi, H., Oetliker, H., Brodbeck, U., Ott, P., Schwendimann, B., Fulpius, B.W. 1983. Reconstitution of pure acetylcholine receptor in phospholipid vesicles and comparison with receptor-rich membranes by the use of a potentiometric dye. $J$. Membrane Biol. 74:75-84

McLaughlin, S.A. 1977. Electrostatic potentials at membranesolution interfaces. Curr. Top. Membr. Transp. 9:71-144

Milsmann, M.H.W., Schwendener, R.A., Weder, H.G. 1978 The preparation of large single bilayer liposomes by a fast and controlled dialysis. Biochim. Biophys, Acta 512:147-155

Oetliker, H. 1980. Studies on the mechanism causing optical excitation-contraction coupling signals in skeletal muscle. $J$. Physiol. (London) 305:26-32

Oetliker, H. 1981. Divalent cation concentration-dependent fluorescence of isolated sarcoplasmic reticulum vesicles stained with indodicarbocyanine. J. Physiol. (London) 318:11-12

Oetliker, H. 1982. An appraisal of the evidence for a sarcoplasmic reticulum membrane potential and its relation to calcium release in skeletal muscle. J. Muscle Res. Cell Motil. 3:247272

Post, R.L., Sen, A.K., Rosenthal, A.S. 1965. A phosphorylated intermediate in adenosine triphosphate-dependent sodium and potassium transport across kidney membranes. J. Biol. Chem. 240:1437-1444

Racker, E., Fisher, L.W. 1975. Reconstitution of an ATP-dependent sodium pump with an ATPase from electric eel and pure phospholipids. Biochem. Biophys. Res. Commun. 67:11441150

Rhoden, V., Goldin, S.M. 1979. Formation of unilamellar lipid vesicles of controllable dimensions by detergent dialysis. Biochemistry 18:4173-4176

Robinson, J.D. 1983. Kinetic analyses and the reaction mechanism of the Na,K-ATPase. In: Structure. Mechanism and Function of the Na/K Pump. J.F. Hoffman and B. Forbush, III, editors. Curr. Top. Membr. Transp. 19:485-512

Robinson, J.D., Flashner, M.S. 1979. The ( $\left.\mathrm{Na}^{+}+\mathrm{K}^{-}\right)$-activated ATPase. Enzymatic and transport properties. Biochim. Biophys. Acta 549:145-176

Ross, W.N., Salzberg, B.M., Cohen, L.B., Grinvald. A., Davila. H.V., Waggoner, A.S., Wang. C.H. 1977. Changes in absorption, fluorescence, dichroism, and birefringence in stained giant axons: Optical measurements of membrane potential. $J$. Membrane Biol. 33:141-183

Schuurmans-Stekhoven, F., Bonting. S.L. 1981. Transport of adenosin triphosphatases: Properties and function. Physiol. Rev. 61:1-76

Schwartz, A., Nagano, K., Nakao, M., Lindenmayer, G.E., Allen, J.C. 1971. The sodium- and potassium-activated adenosinetriphosphatase system. Meth. Pharmacol. 1:361-388

Sims, P.J., Waggoner, A.S., Wang, Ch.H., Hoffman, J.F. 1974. Studies on the mechanism by which cyanine dyes measure membrane potential in red blood cells and phosphatidylcholine vesicles. Biochemistry 13:3315-3329

Skou, J.C. 1975. The $\left(\mathrm{Na}^{+}+\mathrm{K}^{+}\right)$activated enzyme system and its relationship to transport of sodium and potassium. Q. Rev Biophys. 7:401-431

Skriver, E., Maunsbach, A.B., Anner, B.M.. Jørgensen, P.L. $1980 \mathrm{a}$. Electron microscopy of phospholipid vesicles reconstituted with purified renal $\mathrm{Na}, \mathrm{K}-\mathrm{ATPase}$. Cell Biol. Int. Rep. 4:585-591

Skriver, E., Maunsbach, A.B., Jørgensen, P.L. 1980b. Ultrastructure of $\mathrm{Na}$, K-transport vesicles reconstituted with purified renal Na,K-ATPase. J. Cell. Biol. 86:746-754

Stark, G., Benz, R. 1971. The transport of potassium through lipid bilayer membranes by the neutral carriers valinomycin and monactin. Experimental studies to a previously proposed model. J. Membrane Biol. 5:133-153

Waggoner, A.S. 1979. Dye indicators of membrane potential. Annu. Rev. Biophys. Bioeng. 8:47-68

Received 24 August 1984; revised 19 November 1984

\section{Appendix A}

\section{Effects of Vesicle Heterogeneity on the Time Course of Fluorescence Signals}

Since the formation of vesicles and the incorporation of protein into the vesicle membrane are statistical processes, the vesicle population is necessarily heterogenous with respect to size and to the number of pump molecules per vesicle. In order to account for the effects of vesicle heterogeneity on the time course of the fluorescence signal $\Delta F / F_{0}$, we assume that the measured fluorescence intensity is the sum of independent contributions from the single vesicles. If $\Delta f_{j}$ and $f_{j o}$ are the contributions of vesicle $j$ to $\Delta F$ and $F_{0}$, respectively, the relations
$\Delta F=\sum_{j} \Delta f_{j} ; F_{o}=\sum_{j} f_{j o}$

hold. We further assume that the ratio $\Delta f_{j} / f_{j}$, is a unique function of membrane voltage $U$, independent of vesicle size. The following analysis is restricted to experiments in the presence of a high valinomycin concentration in which $U$ is determined by the internal potassium concentration $c_{j} \equiv\left(c_{\mathrm{K}}^{i}\right)_{j}$ of the vesicle according to the Nernst relation [Eq. (1)]. This means that $\Delta f_{j} / f_{j o}$ is a unique function $g\left(c_{j}\right)$ of $c_{j}$ which is determined by the calibration curve (Fig. 2) and the Nernst relation:

$\Delta f_{j} / f_{j o}=g\left(c_{j}\right) \equiv\left(\Delta F / F_{o}\right)_{\text {calibration }}$. 
Furthermore, since $f_{i v}$ and $F_{i}$, refer to the same membrane voltage, the ratio $f_{i v} / F_{0}$ is equal to $A_{t} / A_{\text {, where }} A_{i}$ is the membrane area of vesicle $j$ and $A_{t}$ is the total membrane area of the vesicle suspension. Together with Eqs. (A1) and (A2) this gives

$\frac{\Delta F}{F_{i}} \equiv y(t)=\frac{1}{A_{t}} \sum_{j} A_{i} g\left[c_{i}(t)\right]$.

For the analysis of Eq. (A3) we introduce a statistical model of the vesicle population which is based on the following assumptions:

(a) The probability per unit area of the vesicle membrane for the presence for an outward-oriented pump molecule (ATP-bind ing site facing outward) is constant, independent of the vesicle radius. For a spherical vesicle of external radius $r$ the average number $\bar{n}$ of outward-oriented pumps is then given by:

$\bar{n}=4 \pi r^{2} \chi$

where $\chi$ is the average density $\left(\mu \mathrm{m}^{-2}\right)$ of outward-oriented pumps.

(b) The vesicle radii $r$ are normally distributed with a density function

$\rho(r)=\frac{1}{\sigma \sqrt{2 \pi}} \exp \left[-\left(r-r^{2}\right)^{2} / 2 \sigma^{2}\right]$

$\sigma \equiv \overline{(r-\bar{r})^{2}}$ is the variance of the distribution and $\bar{r}$ the average vesicle radius.

(c) Pump molecules are inserted independently into the vesicle membrane, so that the probability $P_{n}(r)$ that a vesicle of radius $r$ contains exactly $n$ outward-oriented pumps is given by the Poisson relation:

$P_{n}(r)=\frac{\exp (-\bar{n})(\bar{n})^{n}}{n !}$

The assumption of a Poisson distribution for $P_{n}(r)$ is consisten with the electron-microscopic analysis of particle numbers in freeze-fracture preparations of reconstituted ATPase-vesicles (Anner et al., 1984).

For a vesicle of radius $r$ the ratio $A_{i} / A_{t} \equiv A(r) / A$, in Eq. (A3) is equal to $4 \pi r^{2} / N A$ where $N$ is the total number of vesicles and $A$ the average membrane area per vesicle:

$A=4 \pi \int_{0}^{x} r^{2} \rho(r) d r \approx 4 \pi \int_{x}^{x} r^{2} \rho(r) d r=4 \pi\left(r^{2}+\sigma^{2}\right)$.

This gives $A(r) / A_{t}=r^{2} / N\left(\vec{r}^{2}+\sigma^{2}\right)$. The sum in Eq. (A3) may then be replaced by a double sum over $n$ and over $p$ radius intervals $\left(r_{k}\right.$ $\left.-\Delta r / 2, r_{k}+\Delta r / 2\right):$

$y(t) \approx \frac{\Delta r}{\bar{r}^{2}+\sigma^{2}} \sum_{k=1}^{p} \sum_{n=0}^{\infty} r_{k}^{2} P_{n}\left(r_{k}\right) \rho\left(r_{k}\right) g\left[c_{k n}(t)\right]$

$r_{k} \equiv \bar{r}-\Delta r(p+1-2 k) / 2$.

$c_{k n}(t)$ is the $\mathrm{K}^{+}$concentration in a vesicle of radius $r_{k} \pm \Delta r / 2$ containing $n$ outward-oriented pumps. The rate of change of $c_{k n}$ is related to the pump rate $v_{k n}$ and the volume $V_{k}$ of the internal aqueous space of the vesicle according to Eq. (3):

$\frac{d c_{k n}}{d t}=-\frac{\nu n_{k} v_{k n}}{V_{k}}$
The concentration dependence of $v_{k n}$ may be approximately described by the Michaelis-Menten equation:

$v_{k n}=\frac{v_{m} c_{k n}}{c_{k n}+\mathbf{K}}$

Since $\mathrm{K}$ is of the order of $0.1 \mathrm{~mm}$ (Robinson \& Flashner, 1979), much smaller than the initial $\mathrm{K}^{+}$concentration in the vesicle $(c$, $=140 \mathrm{mM})$, the initial pump rate $v_{s}$ is very close to the maximum pump rate $v_{m}$. Equation (A9) and (A 10) together yield $c_{k n}(t)$ in implicit form:

$1-\frac{c_{k n}}{c_{c}}-\frac{\mathbf{K}}{c_{c}} \ln \frac{c_{h n}}{c_{o}}=\frac{t}{\tau_{h n}}$

$\tau_{h n} \equiv \frac{c_{1}, V_{k}}{\nu n_{k} v_{m}}$.

$\tau_{h n}$ is the exchange time of the vesicle, i.e.. the time after which the $\mathrm{K}^{-}$concentration in the vesicle has dropped to nearly zero $\left(c_{1,} \simeq \mathrm{K} \ll c_{1}\right)$.

In order to evaluate the initial pump rate $v_{n} \approx v_{m}$. Eq. (A8) [together with Eqs. (A4)-(A6) and (A11)] was numerically fitted to the experimentally determined function $y(t)$. Values of $\bar{r}$ and $\sigma$ were taken from the light-scattering measurements $(\bar{r}=45 \mathrm{~nm}, \sigma$ $=5 \mathrm{~nm})$, and $\Delta r$ was chosen to be $2.5 \mathrm{~nm}$. The summation in Eq. (A8) was carried out up to terms $\geq 10^{-3} y$. The average surface density $\chi$ which is needed in order to determine $\bar{n}(r)$ may be estimated from the relation

$x=L f \beta \rho_{l} d / M$

where $L$ is Avogadro's constant, $f$ the fraction of outward-oriented pumps, $\beta$ the protein/lipid ratio (wt/wt), $\rho_{l} \simeq 1 \mathrm{~g} / \mathrm{cm}^{3}$ the density of lipid, $d \simeq 5 \mathrm{~nm}$ the membrane thickness and $M=$ $280,000 \mathrm{~g} / \mathrm{mol}$ the molar mass of the protein. An upper limit of $\chi$ is obtained using $f=1$ and $\beta=0.06$ (the protein/lipid ratio prior to dialysis), which yields $\chi=640 \mu \mathrm{m}^{-2}$. Since the efficiency of protein incorporation and the degree of orientation in the membrane is not known, $\chi$ was treated as an adjustable parameter. With $\bar{r}=45 \mathrm{~nm}, \sigma=5 \mathrm{~nm}, \Delta r=2.5 \mathrm{~nm}, \nu=3, c_{,}=140 \mathrm{mM}, \mathrm{K}=$ $0.1 \mathrm{mM}$ and $V_{k}=(4 \pi / 3)\left(r_{k}-d\right)^{3}$, optimum values of $v_{m}$ and $\chi$ were determined which gave the best fit to the experimental $y(t)$ curve, as described in the Results section.

A much simpler analysis is possible when the vesicle radii are so narrowly distributed that $r \equiv \bar{r}$ can be assumed. Under this condition the relation

$\left(\frac{d y}{d t}\right)_{t=0}=-\frac{\nu \bar{n} v_{o}}{V}\left(\frac{d g}{d c}\right)_{c_{t}}$

follows from Eq. (A8), (A11) and (A12) ( $V$ is the volume of the entrapped aqueous space). In this case the initial transport rate $v_{0}$ can be directly determined from the initial rate of change of $y(t)$ when the mean number $\bar{n}$ of outward-oriented pumps is known.

Combining Eq. (A14) with the relation

$\left(\frac{d y}{d t}\right)_{t=0}=\left(\frac{d g}{d c}\right)_{c t}\left(\frac{d c}{d t}\right)_{t=0}$

yields Eq. (3) (with $\bar{n} \equiv n_{\rho}$ ). Thus, Eq. (3) is approximately valid in the limit $t=0$ for vesicles with a narrow size distribution. 